Pure and Applied Mathematics Quarterly

Volume 2, Number 3

(Special Issue: In honor of

Robert MacPherson, Part 1 of 3)

837-865, 2006

\title{
Intersection Numbers in Quasi-Hamiltonian Reduced Spaces
}

\author{
Lisa Jeffrey and Joon-Hyeok Song \\ Dedicated to Bob MacPherson on the occasion of his 60th birthday
}

\begin{abstract}
Jeffrey and Kirwan [15] gave expressions for intersection pairings on the reduced space of a particular Hamiltonian $G$-space in terms of iterated residues. The definition of quasi-Hamiltonian spaces was introduced in [1]. In [2] a localization formula for equivariant de Rham cohomology of a compact quasi-Hamiltonian $G$-space was proved. In this paper we prove a residue formula for intersection pairings of reduced spaces of quasi-Hamiltonian $G$ spaces, by constructing a corresponding Hamiltonian $G$-space. Our formula is a close analogue of the result in [2]. In this article we rely heavily on the methods of [15]; for the general class of compact Lie groups $G$ treated in [2], we rely on results of Szenes and Brion-Vergne concerning diagonal bases.
\end{abstract}

\section{INTRODUCTION}

Alekseev, Meinrenken and Woodward [2] (see also [3]) proved a formula for intersection numbers in quasi-Hamiltonian $G$-spaces, spaces which had been introduced in [1]. Our objective is to prove a close analogue of the formula in [2] modelled on the proof for intersection numbers in moduli spaces of representations of the fundamental group of a Riemann surface [15], using the residue theorem.

Our formula reduces to a sum over components $F$ of the fixed point set of subtori $S$ of $T$ for which $T / S$ acts locally freely on $F$. Our formula is a close analogue of the formula in [2]; in Section 6 we comment on the differences between the two formulas.

Received July 17, 2005. 
We remark that the moduli spaces of interest in [15] are reduced spaces of a quasi-Hamiltonian space, namely the product $G^{2 h}$ where $G=S U(n)$ and $h$ is the genus of the Riemann surface.

The most important new results treated in our article are as follows. First, we observe that the method used in [15] (by the first author and F. Kirwan) applies to quasi-Hamiltonian spaces more generally, not only to $G^{2 h}$. Second, we adapt this method using the diagonal bases of Szenes and Brion-Vergne [8, 9, 21] which enables us to treat quasi-Hamiltonian spaces with an action of any compact Lie group $G$, not only $G=S U(n)$. Finally, our inductive treatment of the fixed point set (Theorem 5.5) means that it is unnecessary to identify the components of the fixed point set at each stage of the induction.

The layout of this article is as follows. In Section 1 we recall the properties of quasi-Hamiltonian $G$-spaces (spaces with group-valued moment maps) and we state Szenes' theorem (which enables one to pass between sums over the weight lattice and iterated residues). Finally we recall the residue theorem which expresses intersection numbers of reduced spaces of Hamiltonian systems in terms of fixed point data on the original Hamiltonian system. In Section 2 we construct the Hamiltonian space corresponding to a given quasi-Hamiltonian space. In Section 3 we summarize material from [15] on equivariant Poincaré duals, and in section 4 we summarize material from [15] on periodicity (an important property of Hamiltonian spaces corresponding to quasi-Hamiltonian spaces).

In Section 5 we prove a residue formula (Theorem 5.5) for intersection numbers in the reduced space of a quasi-Hamiltonian $G$-space (where $G$ is a compact Lie group). In Section 6 we recall the results of Alekseev, Meinrenken and Woodward [2] and give a proof of a close analogue of the main result of [2] by combining Szenes' theorem with our residue formula from Section 5 . We describe the differences between the two formulas. Finally in Section 7 we describe some concrete examples.

The main result of [2] (Theorem 5.2 of that article) is stated in terms of a sum over the dominant weights $\lambda$ of a group $G$ (which are identified with elements of t using an inner product on the Lie algebra of $G$ ) and over the components $F$ of the fixed point set of the vector field $v_{\lambda}$ on $M$ associated to the action of $\lambda$. Theorem 5.2 of [2] is closely related to Theorem 5.5, as we show using Szenes' theorem. We need to use a very special case of Theorem 5.2 of [2] (Proposition 6.5 ), the case when $G$ is a torus acting locally freely on $M$; we expect that this special case may have a proof independent of the proof given in [2].

Our proof of an analogue of Theorem 5.2 of [2] sheds light on a novel feature of quasi-Hamiltonian spaces, namely the components $F$ of the fixed point set of some subtorus $S$ of $T$ for which $T / S$ acts locally freely on $F$. Unlike the case of Hamiltonian $T$-actions, it is possible that the fixed point set $M^{T}$ is empty 
although some connected subgroups $S$ of $T$ have nonempty fixed point sets - an example due to Chris Woodward (treated in Section 7 (Example 7.3)) demonstrates how this situation may arise.

1.1. Group-valued moment maps. Throughout this paper $G$ denotes a compact Lie group of rank $r$ with Lie algebra $\mathbf{g}$. A $G$-manifold is a compact manifold $M$ together with a right action of $G$.

Definition 1.1. For $\xi \in \mathbf{g}$ we denote by $\nu_{\xi}$ the vector field on $M$ generated by $\xi$.

Let $\chi \in \Omega^{3}(G)$ denote the canonical closed bi-invariant 3-form on G:

$$
\chi=\frac{1}{12}\langle\theta,[\theta, \theta]\rangle=\frac{1}{12}\langle\bar{\theta},[\bar{\theta}, \bar{\theta}]\rangle .
$$

Here we have introduced a bi-invariant inner product $\langle\cdot, \cdot\rangle$ on $\mathbf{g}$, and we denote by $\theta$ the left-invariant Maurer-Cartan form, while $\bar{\theta}$ denotes the right-invariant Maurer-Cartan form.

Definition 1.2. [1] A quasi-Hamiltonian $G$-space is a $G$-manifold $M$ together with an invariant 2-form $\omega \in \Omega(M)^{G}$ and an equivariant map $\Phi \in C^{\infty}(M, G)^{G}$ (the quasi-Hamiltonian moment map) such that:

(1) The differential of $\omega$ is given by:

$$
d \omega=-\Phi^{*} \chi
$$

(2) The map $\Phi$ satisfies

$$
\iota\left(\nu_{\xi}\right) \omega=\frac{1}{2} \Phi^{*}\langle\theta+\bar{\theta}, \xi\rangle
$$

(3) At each $x \in M$, the kernel of $\omega_{x}$ is given by

$$
\operatorname{ker} \omega_{x}=\left\{\nu_{\xi}(x), \xi \in \operatorname{ker}\left(A d_{\Phi(x)}+1\right)\right\}
$$

By analogy with Meyer-Marsden-Weinstein reduced spaces, quasi-Hamiltonian reduced spaces are defined in the following way. Let $g \in G$ be a regular value of the quasi-Hamiltonian moment map $\Phi$. The preimage $\Phi^{-1}(g)$ is a smooth manifold on which the action of the centralizer $G_{g}$ is locally free. Then the reduced space $M_{g}=\Phi^{-1}(g) / G_{g}$ is a symplectic orbifold.

Theorem 1.3. (quasi-Hamiltonian reduction, [1]) Let $M$ be a quasi-Hamiltonian $G_{1} \times G_{2}$-space and $g \in G_{1}$ be a regular value of the moment map $\Phi_{1}: M \rightarrow G_{1}$. Then the pull-back of the 2-form $\omega$ to $\Phi_{1}^{-1}(g)$ descends to the reduced space

$$
M_{g}=\Phi_{1}^{-1}(g) / G_{g}
$$


and makes it into a quasi-Hamiltonian $G_{2}$-space. In particular, if $G_{2}=\{e\}$ is trivial, then $M_{g}$ is a symplectic orbifold.

\subsection{Szenes' Theorem.}

1.2.1. General $G$. Let $G$ be a Lie group of rank $r$ with maximal torus $T$. The general version of Szenes' theorem (Theorem 1.13 below) is proved in [21]; a different proof is given in [9] (Theorem 27). We shall require the following definitions.

The fundamental Weyl chamber is denoted $\mathbf{t}_{+}$; it is a fundamental domain for the action of the Weyl group on $\mathbf{t}$.

We define variables $\left\{Y_{j}, j=1, \ldots, r\right\}$ by $Y_{j}=e_{j}(X)$ for $X \in \mathbf{t}$ and $e_{j}$ denoting the simple roots of $G$.

If $g\left(Y_{k}, \ldots, Y_{r}\right)$ is a meromorphic function of $Y_{k}, \ldots, Y_{r}$, we interpret $\operatorname{Res}_{Y_{k}=0} g\left(Y_{k}, \ldots, Y_{r}\right)$ as the ordinary one-variable residue of $g$ regarded as a function of $Y_{k}$ with $Y_{k+1}, \ldots, Y_{r}$ held constant.

Definition 1.4. ([9]) Let $\mathbf{V}$ be a real vector space and let $\triangle \in \mathbf{V}^{*}$ be a finite collection of dual vectors. The set

$$
\mathcal{H}=\cup_{\alpha \in \triangle}\{\alpha=0\}
$$

is called a hyperplane arrangement. An element in $\mathbf{V}$ is called regular if it is not in $\mathcal{H}$.

Remark 1.5. For our purposes $\mathbf{V}=\mathbf{t}$, the Lie algebra of the maximal torus $T$.

Definition 1.6. The ring $R_{\triangle}$ is the set of rational functions with poles on $\mathcal{H}$.

A subset $\sigma$ of $\triangle$ is called a basis of $\triangle$ if the elements $\alpha \in \sigma$ form a basis of $\mathbf{V}$. We denote the set of bases of $\triangle$ by $\mathcal{B}(\triangle)$. An ordered basis is a sequence of elements of $\triangle$ whose underlying set is a basis.

Definition 1.7. If $\sigma$ is a basis of $\triangle$ we let

$$
\phi_{\sigma}(X)=\frac{1}{\prod_{\alpha \in \sigma} \alpha(X)}
$$

and call $\phi_{\sigma}$ a simple fraction. The collection of simple fractions is denoted $S_{\triangle}$.

Definition 1.8. ([9], before Definition 4) When $\tau=\left(\alpha_{i_{1}}, \ldots, \alpha_{i_{r}}\right)$ is an ordered basis of $\mathbf{t}$ and $X \in \mathbf{t} \otimes \mathbb{C}$, , we define variables $Z_{j}^{\tau}=\alpha_{i_{j}}(X)$ for $j=1, \ldots, r$. If $f$ is a meromorphic function on $\mathbf{t} \otimes \mathbb{C}$, then we define

$$
\operatorname{Res}^{\tau}(f)=\operatorname{Res}_{Z_{1}^{\tau}=0} \ldots \operatorname{Res}_{Z_{r}^{\tau}=0}(f)
$$

for $X \in \mathbf{t} \otimes \mathbb{C}$

Remark 1.9. Note that the value of $\operatorname{Res}^{\tau}(f)$ depends on the ordering of the basis $\tau$. 
Definition 1.10. Let $\mathbf{V}$ be a real vector space. A diagonal basis $O B$ is a subset of the ordered bases $\mathcal{O} B(\triangle)$ formed from a collection

$$
\triangle=\left\{\alpha_{1}, \ldots, \alpha_{N}\right\}
$$

of elements of $\mathbf{V}^{*}$ (so that the null spaces of the $\alpha_{j}$ specify a collection of hyperplanes in $\mathbf{V}$ ) satisfying the following conditions:

(1) The set of simple fractions $\phi_{\sigma}$ corresponding to the bases $\sigma \in O B$ forms a basis of $S_{\triangle}$

(2) The collection of meromorphic functions on $\mathbf{V} \otimes \mathbb{C}$ given by

$$
\phi_{\sigma}(X)=\frac{1}{\alpha_{i_{1}}^{\sigma}(X) \ldots \alpha_{i_{r}}^{\sigma}(X)}
$$

(for $\sigma \in O B$ given by $\sigma=\left(\alpha_{i_{1}}^{\sigma}, \ldots, \alpha_{i_{r}}^{\sigma}\right)$ ) satisfies

$$
\operatorname{Res}^{\tau}\left(\phi_{\sigma}\right)=\delta_{\sigma}^{\tau} \text {, }
$$

where $Z_{j}^{\tau}=\alpha_{i_{j}}^{\tau}(X)$ when $\tau=\left(\alpha_{i_{1}}^{\tau}, \ldots, \alpha_{i_{r}}^{\tau}\right)$.

Remark 1.11. Note that a diagonal basis is not a basis for $\mathbf{V}$, but rather a collection of bases for $\mathbf{V}$ (which give a basis for the distinguished subset $S_{\triangle}$ of the meromorphic functions on $\mathbf{V}$ determined by the hyperplanes in $\triangle$ ).

Remark 1.12. ([8], Proposition 14; [21], Proposition 3.4) A total order on $\triangle$ gives rise to a diagonal basis.

Theorem 1.13. [Szenes' theorem] [21, 9] Let $f$ be a meromorphic function on $\mathbf{t} \otimes \mathbb{C}$ with poles only on a collection $\triangle$ of hyperplanes. Let $\mathbf{N}$ be a lattice in $\mathbf{t}$ and $\mathbf{N}_{\text {reg }}$ the regular points in $\mathbf{N}$. Let $\mathbf{M}$ be the lattice dual to $\mathbf{N}$. Let $O B$ be a diagonal basis associated to the hyperplane arrangement $\triangle$. Then

$$
\sum_{n \in \mathbf{N}_{\text {reg }}} e^{<t, 2 \pi i n>} f(2 \pi i n)=\sum_{\sigma \in O B} \operatorname{Res}^{\sigma}\left(f(z) F_{\sigma}^{t}(-z)\right) .
$$

Here, for $\sigma \in O B$ we define

$$
F_{\sigma}^{t}(-z)=\frac{1}{\left|\mathbf{M} / \mathbf{M}_{\sigma}\right|} \sum_{m \in \mathcal{R}(t, \sigma)} \frac{e^{(t-m)(z)}}{\prod_{\alpha \in \sigma}\left(1-e^{\alpha(z)}\right)}
$$

where $\mathcal{R}(t, \sigma)=\left\{u \in \mathbf{M} \mid t-u=\sum_{\alpha \in \sigma} n_{\alpha} \alpha, 0 \leq n_{\alpha}<1\right\}$. It follows that $\mathcal{R}(t, \sigma)$ is in bijective correspondence with $\mathbf{M} / \mathbf{M}_{\sigma}$, where $\mathbf{M}_{\sigma}=\oplus_{\alpha \in \sigma} \mathbb{Z} \alpha \subset \mathbf{M}$.

Remark 1.14. Notice that the left hand side of (1.1) is independent of the choice of diagonal basis $O B$.

Remark 1.15. For our purposes $\mathbf{N}$ is usually the integer lattice $\Lambda^{I} \subset \mathbf{t}$ and $\mathbf{M}$ the weight lattice. 
1.2.2. $G=S U(n)$. The Lie algebra $\mathbf{t}=\mathbf{t}_{n-1}$ of the maximal torus $T$ of $S U(n)$ is

$$
\mathbf{t}=\left\{\left(X_{1}, \ldots, X_{n}\right) \in \mathbb{R}^{n}: X_{1}+\cdots+X_{n}=0\right\}
$$

Define coordinates $Y_{j}=e_{j}(X)=X_{j}-X_{j+1}$ on $\mathbf{t}$ for $j=1, \ldots, n-1$. The positive roots of $S U(n)$ are then $\gamma_{j k}(X)=X_{j}-X_{k}=Y_{j}+\cdots+Y_{k-1}$ for $1 \leq j<k \leq n$. The integer lattice $\Lambda^{I}$ of $S U(n)$ is generated by the simple roots $e_{j}, j=1, \ldots, n-1$. The dual lattice to $\Lambda^{I}$ with respect to the inner product $\langle\cdot, \cdot\rangle$ (in this case the Euclidean inner product on $\mathbb{R}^{n}$ restricted to the hyperplane (1.3)) is the weight lattice $\Lambda^{w} \subset \mathbf{t}$; in terms of the inner product $\langle\cdot, \cdot\rangle$, it is given by $\Lambda^{w}=\{X \in$ $\mathbf{t}: Y_{j} \in \mathbb{Z}$ for $\left.j=1, \ldots, n-1\right\}$. We define also $\Lambda_{\text {reg }}^{w}=\left\{X \in \Lambda^{w}: \gamma_{j k}(X) \neq\right.$ 0 for any $j \neq k\}$.

Definition 1.16. Let $f: \mathbf{t} \otimes \mathbb{C} \rightarrow \mathbb{C}$ be a meromorphic function ${ }^{1}$ of the form

$$
f(X)=g(X) e^{-\gamma(X)}
$$

where $\gamma(X)=\gamma_{1} Y_{1}+\cdots+\gamma_{n-1} Y_{n-1}$ for $\left(\gamma_{1}, \ldots, \gamma_{n-1}\right) \in \mathbb{R}^{n-1}$. We define

$$
[[\gamma]]=\left([[\gamma]]_{1}, \ldots,[[\gamma]]_{n-1}\right)
$$

to be the element of $\mathbb{R}^{n-1}$ for which $0 \leq[[\gamma]]_{j}<1$ for all $j=1, \ldots, n-1$ and $[[\gamma]]=\gamma \bmod \mathbb{Z}^{n-1}$. (In other words, $[[\gamma]]=\sum_{j=1}^{n-1}[[\gamma]]_{j} e_{j}$ is the unique element of $\mathbf{t} \cong \mathbb{R}^{n-1}$ which is in the fundamental domain defined by the simple roots for the translation action on $\mathbf{t}$ of the integer lattice, and which is equivalent to $\gamma$ under translation by the integer lattice.)

We also define the meromorphic function $[[f]]: \mathbf{t} \otimes \mathbb{C} \rightarrow \mathbb{C}$ by

$$
[[f]](X)=g(X) e^{-[[\gamma]](X)} .
$$

Theorem 1.17. [Szenes' theorem for $S U(n)]$ ([15],[21]) Let $f: \mathbf{t} \otimes \mathbb{C} \rightarrow \mathbb{C}$ be a meromorphic function of the form $f(X)=g(X) e^{-\gamma(X)}$ where $\gamma(X)=$ $\gamma_{1} Y_{1}+, \ldots,+\gamma_{n-1} Y_{n-1}$ with $0 \leq \gamma_{n-1}<1$, and $g(X)$ is a rational function of $X$ decaying rapidly at infinity with poles only at the zeros of the roots $\gamma_{j k}$. Then

$$
\sum_{\lambda \in \Lambda_{\text {reg }}^{w}(\mathbf{t})} f(2 \pi i \lambda)=\operatorname{Res}_{Y_{1}=0} \ldots \operatorname{Res}_{Y_{n-1}=0}\left(\frac{\sum_{w \in W_{n-1}}[[w(f)]](X)}{\left(e^{\left.-Y_{n-1}-1\right) \cdots\left(e^{-Y_{1}}-1\right)}\right.}\right)
$$

where $W_{n-1}$ is the Weyl group of $S U(n-1)$ embedded in $S U(n)$ using the first $n-1$ coordinates.

Definition 1.18. Let $c$ be an element of $Z(G)$ (the center of $G$ ).

Remark 1.19. Note that Theorem 1.17 refers to a particular diagonal basis for $S U(n)$ which does not generalize to other Lie groups. See Section 5.3 of [21]. Applying Theorem 1.13 to the case $G=S U(n)$ we obtain an alternative formula for the left hand side of (1.5).

1. We shall use this notation for a particular class of rational functions $g$. 
1.3. Residue formulas for the Hamiltonian space. Let $N$ be a symplectic manifold with a Hamiltonian $G$-action. We denote the moment map by $\mu: N \rightarrow$ $\mathbf{g}^{*}$, and define the symplectic reduced space

$$
N_{\text {red }}=\mu^{-1}(0) / G \text {. }
$$

(Note that $\mu$ will refer to moment maps for Hamiltonian group actions, while $\Phi$ refers to moment maps for quasi-Hamiltonian group actions.) The symplectic form on $N_{\text {red }}$ will be denoted $\omega_{\text {red }}$. We define

$$
\bar{\omega}(X)=\omega+\mu(X)
$$

Then $\bar{\omega} \in \Omega_{G}^{2}(N)$ and it is closed under the Cartan model differential and thus defines an element $[\bar{\omega}] \in H_{G}^{2}(N)$. Here $\Omega_{G}^{2}(N)$ refers to the collection of elements of degree 2 in the Cartan model, while $H_{G}^{2}(N)$ is the equivariant cohomology of $N$ with complex coefficients. See for example [6].

Suppose 0 is a regular value of $\mu$. Then there is a natural map $\kappa: H_{G}^{*}(N) \rightarrow$ $H^{*}\left(N_{\text {red }}\right)$ defined by

$$
\kappa: H_{G}^{*}(N) \rightarrow H_{G}^{*}\left(\mu^{-1}(0)\right) \cong H^{*}\left(N_{r e d}\right) .
$$

It is obviously a ring homomorphism. A similar map is defined for quasi-Hamilto -nian $G$-spaces, which we shall also denote by $\kappa$.

The residue theorem ([14], Theorem 8.1) gives a formula for the evaluation of $\kappa(\eta)$ on the fundamental class of $N_{\text {red }}$. Guillemin and Kalkman and independently Martin have given an alternative version of the residue formula which uses the one-variable result inductively:

Theorem 1.20. ([12] Theorem 3.1; [17]) Suppose $N$ is a compact symplectic manifold acted on by a torus $T$ in a Hamiltonian fashion with moment map $\mu$, and $\eta \in H_{T}^{*}(N)$. The corresponding Kirwan map is denoted by $\kappa_{T}: H_{T}^{*}(N) \rightarrow$ $H^{*}\left(N_{\text {red }}\right)$. Assume 0 is a regular value of $\mu$. Then

$$
\int_{N_{\text {red }}} \kappa_{T}(\eta)=\sum_{N_{1}}^{\prime} \int_{\left(N_{1}\right)_{\text {red }}} \kappa_{T / T_{1}}\left(\operatorname{Res}_{1} \eta\right)
$$

Here, $N_{1}$ is a component of the fixed point set of a circle subgroup $T_{1} \cong S^{1}$ of $T$ (so that $\mu\left(N_{1}\right)$ are critical values of $\mu$ ): it is a symplectic manifold equipped with a Hamiltonian action of $T / T_{1}$ and the natural map $\kappa_{T / T_{1}}: H_{T / T_{1}}^{*}\left(N_{1}\right) \rightarrow$ $H^{*}\left(\left(N_{1}\right)_{\text {red }}\right)$, where $\left(N_{1}\right)_{\text {red }}$ is the reduced space

$$
\frac{\left(\mu_{T / T_{1}}\right)^{-1}(0)}{\left(T / T_{1}\right)}
$$

for the action of $T / T_{1}$ on $N_{1}$. We choose a ray $\lambda$ from 0 to the complement of the moment map image of $N$, and sum over those components $N_{1}$ whose moment 
map image intersects $\lambda$. The map Res $s_{1}: H_{T}^{*}(N) \rightarrow H_{T / T_{1}}^{*}\left(N_{1}\right)$ is defined in [12] (3.6) as

$$
\operatorname{Res}_{1}(\eta)=\operatorname{Res}_{X_{1}=0}\left(\frac{i_{N_{1}}^{*} \eta}{e_{N_{1}}}\right)
$$

where

$$
i_{N_{1}}^{*} \eta \in H_{T}^{*}\left(N_{1}\right)=H_{T / T_{1}}^{*}\left(N_{1}\right) \otimes H_{T_{1}}^{*}
$$

is induced from the inclusion map $i_{N_{1}}: N_{1} \rightarrow N, e_{N_{1}}$ is the equivariant Euler class of the normal bundle to $N_{1}$ in $N$, and $X_{1} \in \mathbf{t}_{1}$ is a basis element for $\mathbf{t}_{1}$. We have introduced the notation $H_{T}^{*}$ to denote $H_{T}^{*}(\mathrm{pt})$.

The notation $\sum^{\prime}$ means the sum over those $T_{1}$ and $N_{1}$ for which a (generic) ray $\lambda$ in $\mathbf{t}^{*}$ from 0 to the complement of $\mu_{T}(N)$ intersects $\mu_{T}\left(N_{1}\right)$. We will use this theorem to allow us to make an inductive argument.

\section{Construction}

Let $M$ be a quasi-Hamiltonian $G$-space, where $G$ is a compact Lie group, with moment map $\Phi: M \rightarrow G$ and $\omega \in \Omega^{2}(M)^{G}$. Recall that in Definition 1.18 we had chosen an element $c \in Z(G)$.

We can construct a corresponding Hamiltonian $G$-space $\widetilde{M}$ as follows. Let $\widetilde{M}=\{(m, \Lambda) \in M \times \mathbf{g} \mid \Phi(m)=c \exp (\Lambda)\}$ and $\mu: M \times \mathbf{g} \rightarrow \mathbf{g}$ is defined by

$$
\mu(m, \Lambda)=-\Lambda \text {. }
$$

Then the following diagram commutes:

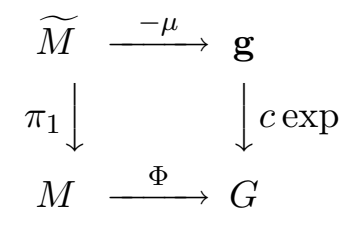

Since $d \exp ^{*} \chi=0$, we can find $\sigma \in \Omega^{2}(\mathbf{g})$ such that $d \sigma=\exp ^{*} \chi$. We see that $d\left(\pi_{1}^{*} \omega-\mu^{*} \sigma\right)=0$. The space $\widetilde{M}$ becomes a Hamiltonian $G$-space with moment map $\mu$ and the invariant 2 -form

$$
\tilde{\omega}=\pi_{1}^{*} \omega-\mu^{*} \sigma \in \Omega^{2}(\widetilde{M})^{G} .
$$

Let $\eta \in H_{G}^{*}(M)$. We want to evaluate

$$
\int_{\Phi^{-1}(c) / G} \kappa\left(\eta e^{\bar{\omega}}\right)
$$


From our construction, we see $\Phi^{-1}(c)=\mu^{-1}(0)$. Therefore

$$
\int_{\Phi^{-1}(c) / G} \kappa\left(\eta e^{\bar{\omega}}\right)=\int_{\mu^{-1}(0) / G} \kappa\left(\eta e^{\bar{\omega}}\right)
$$

(We have assumed $\Phi$ is proper, so these spaces are compact.) So we will evaluate the integral over $\mu^{-1}(0) / G$.

We face the difficulty that $\widetilde{M}$ may be singular. In order to overcome this problem we will use the Poincaré dual introduced in the next section.

\section{Equivariant Poincaré Dual}

Since we know that $M \times \mathbf{g}$ is always smooth, we will work with integration over $M \times \mathbf{g}$, instead of working with integration over $\widetilde{M}$.

Lemma 3.1. ([15] Corollary 5.6) Let $T$ be the maximal torus of $G$ acting on $G$ by conjugation. If $c \in T$ then we can find a T-equivariantly closed differential form $\hat{\alpha} \in \Omega_{T}^{*}(G)$ on $G$ with support arbitrarily close to $c$ such that

$$
\int_{G} \eta \hat{\alpha}=\left.\eta\right|_{c} \in H_{T}^{*}
$$

for all T-equivariantly closed differential forms $\eta \in \Omega_{T}^{*}(G)$.

Remark 3.2. The proof of Lemma 3.1 given in [15] is for the case $G=S U(n)$. Similar arguments apply for general $G$.

Proposition 3.3. ([15] Proposition 5.7) Let $P: M \times \mathbf{g} \rightarrow G$ be defined by

$$
P:(m, \Lambda) \mapsto \Phi(m) \exp (-\Lambda),
$$

and let $c \in T$, so that $\widetilde{M}=P^{-1}(c)$. Let $\alpha=P^{*} \hat{\alpha}$ (where $\hat{\alpha}$ was defined in Lemma 3.1). Hence for $\eta \in \Omega_{G}^{*}(M \times \mathbf{g})$,

$$
\int_{M \times \mathbf{g}} \eta \alpha=\int_{\widetilde{M}} \eta
$$

Definition 3.4. Let $N$ be a Hamiltonian G-manifold with moment map $\mu: N \rightarrow$ $\mathbf{g}^{*}$. We introduce the notation $N / / G$ to denote the symplectic quotient $\mu^{-1}(0) / G{ }^{2}$

We have $\widetilde{M} / / G=\left(P^{-1}(c) \cap \mu^{-1}(0)\right) / G$.

2. This notation is borrowed from algebraic geometry, where it refers to the geometric invariant quotient of a Kähler manifold by the action of a reductive group, which is identified with the symplectic quotient of the maximal compact subgroup. Strictly speaking the notation does not apply to our situation since the objects we work with are not usually Kähler manifolds. 
Let $V$ be a small neighbourhood of $c$ in $G$. In fact, if $V^{\prime}$ is any neighbourhood of $c$ in $T$ containing the closure of $V$ then $^{3}$

$$
\int_{P^{-1}\left(V^{\prime}\right)} \eta \alpha=\int_{\widetilde{M}} \eta \in H_{T}^{*}
$$

Proposition 3.5. (Reduction to the abelian case) [S. Martin [17]] If $T$ is a maximal torus of $G$ and $M$ is a symplectic manifold equipped with an effective Hamiltonian action of $G$, then denote by $\mu: M \rightarrow \mathbf{g}$ the moment map for $G$ and $\mu_{T}: M \rightarrow T$ the $T$ moment map. Assume 0 is a regular value of $\mu$. Then for any regular value $\xi$ of $\mu_{T}$ sufficiently close to 0 and $\eta \in H_{T}^{*}(M)^{W}$ we have that

$$
\begin{gathered}
\int_{\mu^{-1}(0) / G} \kappa^{0}\left(\eta e^{\bar{\omega}}\right)=\frac{n_{1}}{n_{0}^{\prime}|W|} \int_{\mu^{-1}(0) / T} \kappa_{T}^{0}\left(\mathcal{D} \eta e^{\bar{\omega}}\right) \\
=\frac{n_{1}}{n_{0}^{\prime}|W|} \int_{\mu^{-1}(\xi) / T} \kappa_{T}^{\xi}\left(\mathcal{D} \eta e^{\bar{\omega}}\right) \\
=\frac{(-1)^{n_{+}} n_{1}}{n_{0}^{T}|W|} \int_{\mu_{T}^{-1}(\xi) / T} \kappa_{T}^{\xi}\left(\mathcal{D}^{2} \eta e^{\bar{\omega}}\right)
\end{gathered}
$$

where $n_{1}$ is the order of the stabilizer in $G$ of a generic point of $\mu^{-1}(0)$ and $n_{0}^{T}$ (respectively $n_{0}^{\prime}$ ) is the order of the stabilizer in $T$ of a generic point of $\mu_{T}^{-1}(0)$ (respectively $\left.\mu^{-1}(0)\right)$. Here the polynomial $\mathcal{D}: \mathbf{t} \rightarrow \mathbb{R}$ is defined by

$$
\mathcal{D}(X)=\prod_{\gamma>0} \gamma(X)
$$

The equalities (3.2) and (3.3) are valid when the restriction of $\eta$ to $\mu^{-1}(\mathbf{t})$ has compact support, or when $\mu$ is proper. The equality (3.4) is valid provided $\mu_{T}$ is proper.

Remark 3.6. The equality (3.4) is proved in [14], where explicit formulas for the left and right hand sides are proved (establishing the equality).

Remark 3.7. If a symplectic manifold $N$ is acted on by a compact Lie group $G$ with maximal torus $T$, we use the fact that

$$
\mu^{-1}(0)=\mu^{-1}(\mathbf{t}) \cap \mu_{T}^{-1}(0)
$$

to apply Theorem 1.20 when $G$ is nonabelian. Note that $\mu^{-1}(\mathbf{t})$ is smooth because it can be written as $\left(\mu^{\perp}\right)^{-1}(0)$ where $\mu^{\perp}=\pi^{\perp} \circ \mu$ for $\pi^{\perp}: \mathbf{g} \rightarrow \mathbf{t}^{\perp}$ the projection, and we can easily prove $d \mu^{\perp}$ is surjective because $\mu$ is equivariant. Although the hypothesis that $\mu: N \rightarrow \mathbf{g}$ is proper does not imply that $\mu_{T}: N \rightarrow \mathbf{t}$ is proper, it does imply that $\mu_{T}: \mu^{-1}(\mathbf{t}) \rightarrow \mathbf{t}$ is proper.

3. After Lemma 3.10 we shall replace $V$ by a space $\mathcal{V}$ constructed there with $c \in \mathcal{V}$. 
Remark 3.8. By using Proposition 3.5 we can relax the condition from Definition 1.18 that $c \in Z(G)$ to $c \in T$ satisfying a constraint which implies that $\Phi^{-1}(c) / T$ is an orbifold. See Lemma 3.10 below.

Lemma 3.9. (cf. [15], Lemma 5.10) Let $c \in T$ be a regular value of $P$. Then $\left(P^{-1}(V) \cap \mu^{-1}(0)\right) / T$ is an orbifold (for $V$ as above).

Proof. Our first observation is that near $\left(\Phi \circ \pi_{1}\right)^{-1}(c), M$ is endowed with a symplectic structure, since there is a $G$-invariant neighbourhood $V \subset G$ containing $c$ such that the restriction of the closed 2-form $\tilde{\omega}$ (defined in $(2.2))$ to $\left(\Phi \circ \pi_{1}\right)^{-1}(V)$ is nondegenerate. This is true for the following reason. Consider the diagram (2.1). The space $M$ is a smooth manifold, so the space $\widetilde{M}$ is smooth on the preimage under $\Phi \circ \pi_{1}$ of all regular values of $c \exp$ (these regular values contain a neighbourhood $V \subset G$ of $c$ ). The two-form $\tilde{\omega}$ is closed on $\widetilde{M}$. The map $\mu$ satisfies the moment map condition $d \mu_{\xi}=\tilde{\omega}\left(\nu_{\xi}, \cdot\right)$ on $\widetilde{M}$. (See [1] and [13].) Furthermore the 2-form $\tilde{\omega}$ descends under symplectic reduction from $\left(\Phi \circ \pi_{1}\right)^{-1}(V)$ to the standard symplectic form on $\mu^{-1}(0) / G$. It follows that $\tilde{\omega}$ is nondegenerate on $\left(\Phi \circ \pi_{1}\right)^{-1}(V)$, which is an open neighbourhood of $\mu^{-1}(0)$ in $\widetilde{M}$.

We know that $c$ is a regular value for $P: M \times \mathbf{g} \rightarrow G$, and therefore we can choose the neighbourhood $V$ so that all points of $V$ are also regular values of $P$ (by standard properties of the rank of a differentiable map). Because $M \cap \Phi^{-1}(V)$ is symplectic, with moment map $\mu$ related to $\Phi$ as in (2.1), $G$ acts with finite stabilizers at all points of $\left(\Phi \circ \pi_{1}\right)^{-1}(V) \cap \mu^{-1}(0)$. This implies that $T$ also acts with finite stabilizers at all points of $\left(\Phi \circ \pi_{1}\right)^{-1}(V) \cap \mu^{-1}(0)$. Hence $P^{-1}(V) \cap \mu^{-1}(0) / T$ is an orbifold.

We define a one dimensional torus $\hat{T}_{1} \cong S^{1}$ in $G$ generated by a chosen element $\hat{e}_{1}$ in the integer lattice of the Lie algebra of a fixed maximal torus $T$. The corresponding coordinate will be denoted

$$
Y_{1}=\left\langle\hat{e_{1}}, X\right\rangle .
$$

Then $\hat{T}_{1}$ is identified with $S^{1}$ via

$$
e^{2 \pi i t} \in S^{1} \mapsto \exp t \hat{e}_{1} \in \hat{T}_{1}
$$

The one dimensional Lie algebra $\hat{\mathbf{t}}_{1}$ is spanned by $\hat{e}_{1}$. Its orthocomplement in $\mathbf{t}$ (under the inner product) is defined as $\mathbf{t}_{r-1}$. Define $\widetilde{T}_{r-1}$ to be the torus given by $\exp \left(\mathbf{t}_{r-1}\right)$. Also define $T_{r-1}$ to be the quotient torus $T_{r-1}=T / \hat{T}_{1}$, so that its Lie algebra is also $\mathbf{t}_{r-1}$.

Lemma 3.10. (cf. [15], Proposition 6.5) There is a T-invariant open neighbourhood $\mathcal{V} \subset G$ for which the group $T_{r-1}$ acts locally freely on $P^{-1}(\mathcal{V}) \cap \mu^{-1}\left(\hat{\mathbf{t}_{1}}\right)$, so $P^{-1}(\mathcal{V}) \cap \mu^{-1}\left(\hat{\mathbf{t}_{1}}\right) / T_{r-1}$ is an orbifold. 
Proof. Consider the map $P: M \times \mathbf{g} \rightarrow G$ defined at Proposition 3.3. This is a $G$-equivariant map (where $G$ acts on $\mathbf{g}$ and $G$ by the adjoint action).

Let $\mathcal{U}_{r-1} \subset M$ be the subset of $M$ where $T_{r-1}$ acts locally freely. There is an open dense subset $\mathcal{W}$ of $M$ for which open neighbourhoods $\mathcal{W}_{x}$ of $x$ are equipped with Hamiltonian $G$ actions $\mu: \mathcal{W}_{x} \rightarrow$ g. (See [1].)

Let

$$
M_{G}=\{m \in M \mid G \text { acts locally freely at } m\} .
$$

This set is $T$ invariant.

We note that $d \Phi: T_{x} M_{G} \rightarrow T_{\Phi(x)} G$ is surjective for all $x \in M_{G}$, and by the inverse function theorem this implies $\Phi\left(M_{G}\right)$ is an open subset of $G$.

Hence $P\left(M_{G} \times \mathbf{t}_{1}\right)$ is also an open subset of $G$.

We define $\mathcal{V}=P\left(M_{G} \times \mathbf{t}_{1}\right)$. This is a $T$-invariant open set. From now on, without loss of generality we replace our earlier open neighbourhood $V \subset G$ (introduced after Definition 3.4) by $\mathcal{V}$, and we redefine $c$ to be a point in $\mathcal{V}$.

We extend the definition of the composition

$$
\kappa: H_{T}^{*}\left(P^{-1}(c)\right) \rightarrow H_{T}^{*}\left(P^{-1}(c) \cap \mu^{-1}(0)\right) \cong H^{*}\left(P^{-1}(c) \cap \mu^{-1}(0) / T\right)
$$

to

$$
\left.\kappa: H_{T}^{*}\left(P^{-1}(\mathcal{V})\right) \rightarrow H_{T}^{*}\left(P^{-1}(\mathcal{V}) \cap \mu^{-1}(0)\right) \cong H^{*}\left(P^{-1}(\mathcal{V}) \cap \mu^{-1}(0)\right) / T\right) .
$$

By Proposition 3.3 we have

$$
\int_{P^{-1}(c) \cap \mu^{-1}(0) / T} \kappa(\eta)=\int_{P^{-1}(\mathcal{V}) \cap \mu^{-1}(0) / T} \kappa(\eta \alpha)
$$

where $\eta \in H_{G}^{*}(M)$. The class $\alpha$ is the Poincaré dual of $P^{-1}(c)$ in $P^{-1}(\mathcal{V})$.

\section{PERIODICITY}

The space $\widetilde{M}$ is noncompact, so many standard results in symplectic geometry cannot be applied without further analysis. To handle the difficulties of noncompactness we rely on the fact that the moment map $\mu$ is proper (because $\Phi$ is proper and $\left.\mu^{-1}(U)=\Phi^{-1}(\exp U)\right)$ and use a relative version of GuilleminKalkman's theorem (Proposition 4.3) which is valid provided the inverse image of a closed interval under the moment map for a circle action is compact. Using Proposition 4.3 we reduce to the components of the fixed point set of the circle action for which the moment map takes values in a closed interval. Since the moment map for this circle action is proper there are only finitely many components 
of the fixed point set whose moment map takes values in this closed interval, so the sums in Proposition 4.3 and Lemma 4.4 are finite sums.

Proposition 4.1. ([15], Proposition 6.3;[11]) For any symplectic manifold $M$ equipped with a Hamiltonian action of $T=T_{r}$ such that $\widetilde{T}_{r-1}$ acts locally freely on $\mu_{\widetilde{T}_{r-1}}^{-1}(0)$, the symplectic quotient $\mu_{T_{r}}^{-1}(0) / T$ may be identified with the symplectic quotient of $\mu_{\widetilde{T}_{r-1}}^{-1}(0) / \widetilde{T}_{r-1}$ by the induced Hamiltonian action of $\hat{T}_{1}$. Moreover if in addition $T_{r}$ acts locally freely on $\mu_{T_{r}}^{-1}(0)$ then the ring homomorphism $\kappa$ : $H_{T_{r}}^{*}(M) \rightarrow H^{*}\left(\mu_{T_{r}}^{-1}(0) / T_{r}\right)$ factors as

$$
\kappa=\hat{\kappa}_{1} \circ \kappa_{r-1}
$$

where

$$
\kappa_{r-1}: H_{T_{r}}^{*}(M) \rightarrow H_{T_{r}}^{*}\left(\mu_{\widetilde{T}_{r-1}}^{-1}(0)\right) \cong H_{\widehat{T}_{1} \times \widetilde{T}_{r-1}}^{*}\left(\mu_{\widetilde{T}_{r-1}}^{-1}(0)\right) \cong H_{\hat{T}_{1}}^{*}\left(\mu_{\widetilde{T}_{r-1}}^{-1}(0) / \widetilde{T}_{r-1}\right)
$$

and

$\hat{\kappa}_{1}: H_{\hat{T}_{1}}^{*}\left(\mu_{\widetilde{T}_{r-1}}^{-1}(0) / \widetilde{T}_{r-1}\right) \rightarrow H^{*}\left(\left(\mu_{\widetilde{T}_{r-1}}^{-1}(0) \cap \mu_{\hat{T}_{1}}^{-1}(0)\right) /\left(\widetilde{T}_{r-1} \times \hat{T}_{1}\right)\right) \cong H^{*}\left(\mu_{T_{r}}^{-1}(0) / T_{r}\right)$ are the corresponding compositions of restriction maps with similar isomorphisms (i.e., Kirwan maps).

Remark 4.2. Let $T_{r}$ and $T_{r-1}$ be as above Proposition 4.1. Note also that

$$
\kappa=\hat{\kappa}_{r-1} \circ \kappa_{1}
$$

where

$$
\kappa_{1}: H_{T}^{*}(M) \rightarrow H_{\left(T / \hat{T}_{1}\right)}^{*}\left(\mu_{\hat{T}_{1}}^{-1}(0) / \hat{T}_{1}\right)
$$

and

$$
\hat{\kappa}_{r-1}: H_{\left(T / \hat{T}_{1}\right)}^{*}\left(\mu_{\hat{T}_{1}}^{-1}(0) / \hat{T}_{1}\right) \rightarrow H_{T}^{*}\left(\mu_{T}^{-1}(0)\right) \cong H^{*}\left(\mu_{T}^{-1}(0) / T\right)
$$

are defined in a way similar to the definitions of $\hat{\kappa}_{1}$ and $\kappa_{r-1}$ given in the statement of the previous proposition.

We introduce the notation $\kappa^{0}: H_{G}^{*}(M) \rightarrow H^{*}\left(\mu^{-1}(0) / G\right)$ to mean the composition of the restriction map $H_{G}^{*}(M) \rightarrow H_{G}^{*}\left(\mu^{-1}(0)\right)$ and the isomorphism $H_{G}^{*}\left(\mu^{-1}(0)\right) \cong H^{*}\left(\mu^{-1}(0) / G\right)$. The $\operatorname{map} \kappa_{T}^{0}: H_{T}^{*}(M) \rightarrow H^{*}\left(\mu_{T}^{-1}(0) / T\right)$ is defined similarly, and in the same way $\kappa_{T}^{0}: H_{T}^{*}(M) \rightarrow H_{T}^{*}\left(M \cap \mu^{-1}(\mathbf{t})\right) \rightarrow$ $H^{*}\left(M \cap \mu^{-1}(0) / T\right)\left(\right.$ since $\left.\mu^{-1}(0)=\mu_{T}^{-1}(0) \cap \mu^{-1}(\mathbf{t})\right)$. The maps $\kappa_{T}^{\xi}$ are defined in the same way, except that $\mu^{-1}(0)$ is replaced by $\mu^{-1}(\xi)$.

Proposition 4.3. (Dependence of symplectic quotients on parameters) [Guillemin-Kalkman [12]; S. Martin [17]] Let $M$ be a Hamiltonian T-space 
for which the moment map $\mu_{T}$ is proper. If $T=U(1)$ and $n_{0}^{T}$ is the order of the stabilizer in $T$ of a generic point of $\mu_{T}^{-1}(0)$ then

$$
\begin{aligned}
\int_{\mu_{T}^{-1}\left(\xi_{1}\right) / T} \kappa_{T}^{\xi_{1}}\left(\eta e^{\bar{\omega}}\right)-\int_{\mu_{T}^{-1}\left(\xi_{0}\right) / T} \kappa_{T}^{\xi_{0}}\left(\eta e^{\bar{\omega}}\right) & \\
& =n_{0}^{T} \sum_{E \in \mathcal{E}: \xi_{0}<\mu_{T}(E)<\xi_{1}} \operatorname{Res}_{X=0} e^{\mu_{T}(E) X} \int_{E} \frac{\eta(X) e^{\omega}}{e_{E}(X)}
\end{aligned}
$$

where $X \in \mathbb{C}$ has been identified with $2 \pi i X \in \mathbf{t} \otimes \mathbb{C}$ and $\xi_{0}<\xi_{1}$ are two regular values of the moment map. Here $\mathcal{E}$ is the set of all components $E$ of the fixed point set of $T$ on $M$.

Lemma 4.4. (cf. [15] Lemma 6.7) Let $M$ be a quasi-Hamiltonian G-space for which $\Phi$ is proper, ${ }^{4}$ and let the space $\widetilde{M}$ be defined by (2.1). Suppose $\eta \in H_{G}^{*}(M)$. If $\mathcal{V}$ is as defined in the proof of Lemma 3.10, and we define $M=P^{-1}(c)$ for some $c$ in $\mathcal{V}$ so that (by Lemma 3.10) $P^{-1}(\mathcal{V}) \cap \mu^{-1}\left(\hat{\mathbf{t}}_{1}\right) / T_{r-1}$ is an orbifold and we define $N(\mathcal{V})=P^{-1}(\mathcal{V}) \cap \mu^{-1}(0) / T$, then this hypothesis implies also that 0 is a regular value of $\mu_{T / \hat{T}_{1}}: E \rightarrow \mathbf{t} / \hat{\mathbf{t}_{1}}$ for all $E \in \mathcal{E}$, where $\mathcal{E}$ is the set of components of the fixed point set of the action of $\hat{T}_{1}$ on $M \times \hat{\mathbf{t}_{1}}$. We then have that

$$
\begin{gathered}
\int_{N(\mathcal{V})} \kappa\left(\eta e^{\bar{\omega}} e^{-Y_{1}} \alpha\right)=\int_{P^{-1}(\mathcal{V}) \cap \mu^{-1}\left(\hat{e}_{1}\right) / T} \kappa\left(\eta e^{\bar{\omega}} \alpha\right) \\
(4.4)=\int_{N(\mathcal{V})} \kappa\left(\eta e^{\bar{\omega}} \alpha\right)-n_{0} \sum_{E \in \mathcal{E}:-\left\|\hat{e}_{1}\right\|^{2}<\left\langle\hat{e}_{1}, \mu(E)\right\rangle<0} \int_{E / / \widetilde{T}_{r-1}} \kappa_{T / \hat{T}_{1}} \operatorname{Res}_{Y_{1}=0} \frac{\eta e^{\bar{\omega}} \alpha}{e_{E}} .
\end{gathered}
$$

Here $e_{E}$ is the $\hat{T}_{1}$-equivariant Euler class of the normal bundle to $E$ in $\widetilde{M}$, while $n_{0}$ is the order of the subgroup of $\hat{T}_{1} /\left(\hat{T}_{1} \cap \widetilde{T}_{r-1}\right)$ that acts trivially on $P^{-1}(\mathcal{V}) \cap \mu^{-1}\left(\hat{\mathbf{t}_{\mathbf{1}}}\right)$, and $Y_{1}$ was defined in (3.6). Also $\alpha$ is the T-equivariantly closed differential form on $M \times \mathbf{t}$ given by Proposition 3.3 which represents the equivariant Poincaré dual of $\widetilde{M}$, chosen so that the support of $\alpha$ is contained in $P^{-1}(\mathcal{V})$

Proof. Note that throughout this proof we make extensive use of Remark 3.7. Since $\mu^{-1}\left(\hat{\mathbf{t}}_{1}\right)=M \times \hat{\mathbf{t}}_{1}$ is contained in $\mu_{\widetilde{T}_{r-1}}^{-1}(0)$, it follows from Lemma 3.10 that $P^{-1}(\mathcal{V}) \cap \mu^{-1}\left(\hat{\mathbf{t}}_{1}\right) / \widetilde{T}_{r-1}$ is an orbifold. (Here we let $\mu_{\hat{T}_{1}}=\pi_{\hat{\mathbf{t}}_{1}} \circ \mu$, and $\pi_{\hat{\mathbf{t}}_{1}}: \mathbf{t} \rightarrow \hat{\mathbf{t}}_{1}$ is the projection corresponding to the bi-invariant inner product.) The restriction of $\mu_{\hat{T}_{1}}$ to $\mu^{-1}\left(\hat{\mathbf{t}}_{\mathbf{1}}\right)$ is proper, because if $C \subset \hat{\mathbf{t}}_{1}$ is compact, then

$$
\mu_{\hat{T}_{1}}^{-1}(C)=\{(x, \Lambda) \in M \times C: \Phi(x)=\exp (\Lambda)\} .
$$

4. The hypothesis that $\Phi$ is proper is satisfied in many examples, including all compact examples. 
This is compact because $C$ is covered by finitely many compact subsets $U$ of $\hat{\mathbf{t}}_{1}$ on each of which the restriction of the exponential map to $\hat{\mathbf{t}}_{1}$ is a diffeomorphism, and on each $U$ we find that $\{(x, \Lambda) \in M \times U: \Phi(x)=\exp (\Lambda)\}=\Phi^{-1}(\exp U)$, which is compact because $\Phi$ is proper. Also, the support of $\alpha$ is contained in $P^{-1}(\mathcal{V})$. To apply Proposition 4.3 we need that 0 and $\hat{e_{1}}$ are regular values of $\mu_{T}: \mu^{-1}(\mathbf{t}) \rightarrow \mathbf{t}$. This follows from Lemma 3.9 where it is shown that $N(\mathcal{V})$ is an orbifold. (Note that by definition $N(\mathcal{V})$ is identical to $P^{-1}(\mathcal{V}) \cap \mu^{-1}\left(\hat{e_{1}}\right) / T_{r}$.)

Hence Guillemin and Kalkman's proof of Proposition 4.3 can be applied to the $\hat{T}_{1}$-invariant function induced by $\mu_{\hat{T}_{1}}$ on $\widetilde{M} \cap \mu^{-1}(\mathbf{t})$ and the $\hat{T}_{1}$-equivariant form induced by $\eta e^{\bar{\omega}} \alpha$. By combining this with Remark 4.2 and using Theorem 1.20, Remark 3.7 and (3.2) of Proposition 3.5 we get

$$
\begin{aligned}
& \int_{N(\mathcal{V})} \kappa\left(\eta e^{\bar{\omega}} \alpha\right)-\int_{P^{-1}(\mathcal{V})^{T} \cap \mu^{-1}\left(\hat{e}_{1}\right) / T} \kappa\left(\eta e^{\bar{\omega}} \alpha\right) \\
= & n_{0} \sum_{E \in \mathcal{E}:-\left\|\hat{e}_{1}\right\|^{2}<\left\langle\hat{e}_{1}, \mu(E)\right\rangle<0} \int_{E / / T_{r-1}} \operatorname{Res}_{Y_{1}=0} \kappa_{r-1} \frac{\left(\eta e^{\bar{\omega}} \alpha\right)}{e_{E}}
\end{aligned}
$$

Now we need to show (4.3). Note that the restriction of $P: M \times \mathbf{g} \rightarrow G$ to $\mu^{-1}(\mathbf{t})=M \times \mathbf{t}$ is invariant under the translation $s_{\Lambda_{0}}: M \times \mathbf{g} \rightarrow M \times \mathbf{g}$ defined by

$$
s_{\Lambda_{0}}:(m, \Lambda) \mapsto\left(m, \Lambda+\Lambda_{0}\right)
$$

for $\Lambda_{0} \in \Lambda^{I}=\operatorname{ker}(\exp )$ in t. So for $\Lambda_{0}=\hat{e_{1}}$

$$
\begin{aligned}
\int_{P^{-1}(\mathcal{V})^{T} \cap \mu^{-1}\left(\hat{e}_{1}\right) / T_{r}} \kappa\left(\eta e^{\bar{\omega}} \alpha\right) & =\int_{N(\mathcal{V})} \kappa\left(s_{\hat{e}_{1}}^{*}\left(\eta e^{\bar{\omega}} \alpha\right)\right) \\
& =\int_{N(\mathcal{V})} \kappa\left(\eta e^{\bar{\omega}} e^{-Y_{1}} \alpha\right)
\end{aligned}
$$

This proves (4.3).

It is proved in [12] (Proof of (3.10)) that

$$
\kappa_{T / \hat{T}_{1}} \operatorname{Res}_{1}=\operatorname{Res}_{1} \kappa_{T / \hat{T}_{1}}
$$

where the notation $R_{e} s_{1}$ was introduced in (1.8) and on the left hand side of (4.6) we have

$$
\operatorname{Res}_{1}: H_{T}^{*}\left(\mu^{-1}(\mathbf{t}) \cap \widetilde{M}\right) \rightarrow H_{T / \hat{T}_{1}}^{*}\left(\widetilde{M}_{1} \cap \mu^{-1}(\mathbf{t})\right)
$$

(where $\widetilde{M}_{1}$ is the fixed point set of the action of $\hat{T}_{1}$ on $\widetilde{M}$ ) while on the right hand side we have

$$
\operatorname{Res}_{1}: H_{T_{1}}^{*}\left(\mu^{-1}(\mathbf{t}) / /\left(T / T_{1}\right)\right) \rightarrow H^{*}\left(\mu^{-1}(\mathbf{t}) / / T\right) .
$$

This enables us to deduce (4.4) from (4.5). 
Remark 4.5. The proof of Lemma 6.7 in [15] was for the case $M=G^{2 h}$, but the only property of this space used is that $\Phi$ is proper and the explicit identification of a suitable set $\mathcal{V}$ in [15], Proposition 6.5.

We now come to a lemma which asserts that the fixed point set of the action of a circle subgroup on a quasi-Hamiltonian $G$-space is also a quasi-Hamiltonian space. This result is a special case of [4], Proposition 4.4. The result is valid for subgroups $S$ of rank higher than 1 , but we state it only for $S \cong S^{1}$ since this is the only case we need to make the induction work. We include a proof for completeness.

Lemma 4.6. Let $(M, \omega, \mu)$ be a quasi-Hamiltonian $G$-space and let $\hat{T}_{1}=S \cong S^{1}$ be a subgroup of $G$. If $H$ is the fixed point set of the adjoint action of $S$ on $G$ (the subgroup of $G$ consisting of all elements which commute with all elements of $S)$, then $H$ is a Lie subgroup of $G$ and $M^{S}$ is a quasi-Hamiltonian $H$-space.

Proof. Since $M$ is a quasi-Hamiltonian $G$-space, it satisfies the three axioms given in Definition 1.1. We need to check that these axioms are also valid for the $H$-space $M^{S}$.

(1) Let $\iota_{S}: M^{S} \rightarrow M$, and $\Phi_{H}=\left.\Phi_{G}\right|_{M^{S}}$. (Notice that on $M^{S} \Phi_{G}$ takes values in $H$, by equivariance of $\Phi_{G}$.) Then

$$
\begin{aligned}
d \iota_{S}^{*} \omega & =\iota_{S}^{*} d \omega \\
& =-i_{S}^{*} \Phi_{G}^{*} \chi_{G} \\
& =-\Phi_{H}^{*} \chi_{H}
\end{aligned}
$$

(2) If $\xi \in \mathbf{h}$

$$
\begin{aligned}
\iota\left(\nu_{\xi}\right) \iota_{S}^{*} \omega & =\frac{1}{2} \iota_{S}^{*} \Phi_{G}^{*}\left\langle\theta_{G}+\bar{\theta}_{G}, \xi\right\rangle \\
& =\frac{1}{2} \Phi_{H}^{*}\left\langle\theta_{H}+\bar{\theta}_{H}, \xi\right\rangle
\end{aligned}
$$

(3) At each $x \in M^{S}$ and for $\xi \in \mathbf{h}$, we need to show that the kernel of $\iota_{S}^{*} \omega_{x}$ is given by

$$
\operatorname{ker} \iota_{S}^{*} \omega_{x}=\left\{\nu_{\xi}(x), \xi \in \operatorname{ker}\left(A d_{\Phi_{H}(x)}+1\right)\right\} .
$$

We know that for $x \in M^{S}$, $\operatorname{Ker} \omega_{x}=\left\{\nu_{\xi}, \xi \in \mathbf{g}, \operatorname{Ad}_{\Phi(x)} \xi=-\xi\right\}$. If $v \in T_{x} M^{S}$ satisfies $\omega(v, Y)=0$ for all $Y \in T_{x} M$ then $v=\nu_{\xi}$ for some $\xi \in \mathbf{g}$ with $\operatorname{Ad}_{\Phi(x)} \xi=-\xi$. It suffices to prove that $\nu_{\xi}(x) \in T_{x} M^{S}$ if and only if $\xi \in \mathbf{h}$. It is obvious that if $\xi \in \mathbf{h}$ then $\nu_{\xi}(x) \in T_{x} M^{S}$. To prove the converse, we see that if $\xi \notin \mathbf{h}$ then (restricting to $\xi$ which are orthogonal to the Lie algebra of $\operatorname{Stab}(x)$ in the biinvariant inner product, so that the map $\xi \mapsto \nu_{\xi}(x)$ becomes bijective) $\operatorname{Ad}_{s} \xi \neq \xi$ for $s$ a generator of $S$. Hence $\operatorname{Ad}_{s} \nu_{\xi} \neq \nu_{\xi}$ which implies $\nu_{\xi} \notin T_{x} M^{S}$, as we wished to prove. 
Remark 4.7. If $T$ is a torus which is a subgroup of $G$ and $M$ a quasi-Hamiltonian $G$-space, then $\Phi^{-1}(T)$ is a quasi-Hamiltonian T-space. Thus also $\mu^{-1}(\mathbf{t})$ is a Hamiltonian T-space. This is proved in [1].

Remark 4.8. Under the hypotheses of Lemma 4.6, $T \subset H$ and $\hat{T}_{1} \subset Z(H)$. Thus $T_{r-1}=T / \hat{T}_{1}$ is a group of rank $r-1$ with a quasi-Hamiltonian action on $M^{\hat{T}_{1}}$. This enables us to perform an inductive argument.

From Lemma 4.4, we get

$$
\begin{aligned}
& \int_{N(\mathcal{V})} \kappa\left(\eta e^{\bar{\omega}} \alpha\right)-\int_{N(\mathcal{V})} \kappa\left(\eta e^{\bar{\omega}} e^{Y_{1}} \alpha\right)=\int_{N(\mathcal{V})} \kappa\left(\eta e^{\bar{\omega}}\left(1-e^{Y_{1}}\right) \alpha\right) \\
& =n_{0} \sum_{E \in \mathcal{E}:-\left\|\hat{e}_{1} \mid\right\|^{2}<\left\langle\hat{e}_{1}, \mu(E)\right\rangle<0} \int_{E / / T_{r-1}} \kappa_{r-1} \operatorname{Res}_{Y_{1}=0} \frac{\eta e^{\bar{\omega}} \alpha}{e_{E}}
\end{aligned}
$$

Thus

Proposition 4.9. Under the hypotheses and in the notation of Lemma 4.4, we have

$$
(4.9) \int_{N(\mathcal{V})} \kappa\left(\eta e^{\bar{\omega}} \alpha\right)=n_{0} \sum_{E \in \mathcal{E}:-\left\|\hat{e}_{1}\right\|^{2}<\left\langle\hat{e}_{1}, \mu(E)\right\rangle<0} \int_{E / / T_{r-1}} \kappa_{r-1} \operatorname{Res}_{Y_{1}=0} \frac{\left(\eta e^{\bar{\omega}} \alpha\right)}{e_{E}\left(1-e^{Y_{1}}\right)}
$$

\section{Residue FORMUla FOR QUASI-Hamiltonian SPACES}

We shall now list some properties of diagonal bases which will enable us to use the general form of Szenes' theorem (Theorem 1.13) to relate our results with Alekseev-Meinrenken-Woodward's results in Section 6 .

Let $\hat{\mathbf{t}_{1}}$ be the Lie algebra of a circle subgroup $\hat{T}_{1} \cong S^{1}$. Let $\hat{e}_{1}$ be an element of the weight lattice of $T$, which projects to a generator of $\hat{\mathbf{t}}_{1}{ }^{*}$. We will study diagonal bases for $\mathbf{t} \otimes \mathbb{C}$; these will correspond to a choice of weights. We refer to Definition 1.10 and Remarks 1.11 and 1.12 .

Let $S$ be a subgroup of $T$. The purpose of the following lemma is to show that diagonal bases for hyperplane arrangements for the Lie algebra of $S / \hat{T}_{1}$ may be converted into diagonal bases for a hyperplane arrangement in the Lie algebra of $S$ by adjoining a generator of $\hat{\mathbf{t}_{1}}$ to each ordered basis of $\operatorname{Lie}\left(S / \hat{T}_{1}\right)$.

Lemma 5.1. Let $\left\{\alpha_{1}^{\prime}, \ldots, \alpha_{N_{1}}^{\prime}\right\}$ be a collection of elements in $\mathfrak{s}^{*}$, where $S \triangleleft T$ is a subgroup of $T$ such that $\hat{T}_{1} \subset S$, and $\mathfrak{s}$ is the Lie algebra of $S$. Suppose $\sigma^{\prime}=\left\{\sigma_{1}^{\prime}, \ldots, \sigma_{N_{2}}^{\prime}\right\}$ is a diagonal basis for a hyperplane arrangement $\triangle^{\prime}$ in $\mathfrak{s} / \hat{\mathbf{t}_{1}}$ corresponding to a chosen total ordering on the hyperplane arrangement $\triangle^{\prime}$ which 
comprises the weights for the action of $S / \hat{T}_{1}$ on all tangent spaces $T_{F} M_{1}$ for all components $F$ of the fixed point set of the action of $S / \hat{T}_{1}$ on $M_{1}$ for some subgroup $S$ of $T$ satisfying $\hat{T}_{1} \subset S$, where $M_{1}$ is a component of $M^{\hat{T}_{1}}$. So each $\sigma_{j}^{\prime}$ is a basis for $\mathfrak{s} / \hat{\mathbf{t}_{1}}$. Then $\sigma=\left\{\sigma_{1}^{\prime} \cup \hat{e_{1}}, \ldots, \sigma_{N_{2}}^{\prime} \cup \hat{e_{1}}\right\}$ is a diagonal basis for the hyperplane arrangement $\triangle^{\prime} \cup\left\{\hat{e_{1}}\right\}$ in $\mathfrak{s}$, which corresponds to a subset of the weights of the action of $S$ on all tangent spaces $T_{F} M$ at all components $F$ of the fixed point set of the action of $S$ on $M$.

Proof. Suppose $\sigma_{j}^{\prime}=\left(\alpha_{j_{1}}^{\prime}, \ldots, \alpha_{j_{\ell-1}}^{\prime}\right)$ and $\sigma_{k}^{\prime}=\left(\alpha_{k_{1}}^{\prime}, \ldots, \alpha_{k_{\ell-1}}^{\prime}\right)$ are ordered bases which are members of a diagonal basis for a hyperplane arrangement in $\mathfrak{s} / \hat{\mathbf{t}}_{1}$. (Here, $\ell$ is the rank of $S$, and $k=\left(k_{1}, \ldots, k_{\ell-1}\right)$ and $j=\left(j_{1}, \ldots, j_{\ell-1}\right)$ are multi-indices.) We note that if $\sigma_{j}^{\prime}=\left(\alpha_{j_{1}}^{\prime}, \ldots, \alpha_{j_{\ell-1}}^{\prime}\right)$ and $\left(Z^{\prime}\right)_{t}^{\sigma_{j}^{\prime}}=\alpha_{j_{t}}^{\prime}(X)$ for $1 \leq t \leq \ell-1$, then by the definition of a diagonal basis (Definition 1.10, (2)) we have in the notation of Definition 1.10

$$
\operatorname{Res}^{\sigma_{j}^{\prime}}\left(\frac{1}{\alpha_{k_{1}}^{\prime}(X) \ldots \alpha_{k_{\ell-1}}^{\prime}(X)}\right)=\delta_{k}^{j},
$$

where $\delta_{k}^{j}=1$ if all the multi-indices $k_{s}=j_{s}($ for $s=1, \ldots, \ell-1)$ and $\delta_{k}^{j}=0$ otherwise. Then we form

$$
Z_{t}^{\sigma_{j}}=\left(Z^{\prime}\right)_{t}^{\sigma_{j}^{\prime}}, 1 \leq t \leq \ell-1
$$

and $Z_{\ell}^{\sigma_{j}}=\hat{e_{1}}(X)$. (Note that the $Z_{\ell}^{\sigma_{j}}$ are all equal for all $j$.)

Then it is clear that for each $j$ the set which is the union of $\hat{e_{1}}$ and the basis $\sigma_{j}^{\prime}$ is a basis for $\mathfrak{s}$. Also, we have that

$$
\begin{gathered}
\operatorname{Res}^{\sigma_{j}}\left(\frac{1}{\prod_{t=1}^{\ell} Z_{t}^{\sigma_{k}}}\right) \\
=\left(\operatorname{Res}^{\sigma_{j}^{\prime}}\right) \operatorname{Res}_{Z_{\ell}^{\sigma_{j}}=0} \frac{1}{Z_{\ell}^{\sigma_{j}} \prod_{t=1}^{\ell-1}\left(Z^{\prime}\right)_{t}^{\sigma_{k}^{\prime}}} \\
=\operatorname{Res}^{\sigma_{j}^{\prime}} \frac{1}{\prod_{t=1}^{\ell-1}\left(Z^{\prime}\right)_{t}^{\sigma_{k}^{\prime}}}=\delta_{j}^{k}(\text { by }(5.1)) .
\end{gathered}
$$

Hence $\sigma$ defined as in the statement of Lemma 5.1 is a diagonal basis for $\triangle^{\prime} \cup\left\{\hat{e_{1}}\right\}$.

Note that in this section we identify weights of $S$ with weights of $T$ using an invariant inner product on $\mathbf{t}$.

Lemma 5.2. We have

$$
\mathbf{M} / \mathbf{M}_{\sigma}=\mathbf{M}^{\prime} / \mathbf{M}_{\sigma^{\prime}}^{\prime}
$$

where $\mathbf{M}^{\prime}$ is the dual lattice of the lattice $\mathbf{N}^{\prime}$ associated to the hyperplane arrangement $\triangle^{\prime}$ in Lemma 5.1, and $\mathbf{M}_{\sigma^{\prime}}^{\prime}$ is the sublattice of $\mathbf{M}^{\prime}$ corresponding to 
the ordered basis $\sigma^{\prime}$ for $\mathfrak{s} / \hat{\mathbf{t}_{1}}$, and $\sigma=\sigma^{\prime} \cup\left\{\hat{e_{1}}\right\}$ is an ordered basis for $\mathfrak{s}$. Recall that $\mathbf{M}_{\sigma}$ was introduced in the statement of Theorem 1.13.

Proof. By definition $\mathbf{M}$ is the lattice generated by $\mathbf{M}^{\prime}$ and $\hat{e_{1}}$ and $\mathbf{M}_{\sigma^{\prime}}^{\prime}$ is the lattice generated by all $\alpha \in \sigma^{\prime}$ so $\mathbf{M}_{\sigma}$ is the lattice generated by the $\alpha \in \sigma^{\prime}$ together with $\hat{e_{1}}$. Thus the lemma follows immediately.

Lemmas 5.1 and 5.2 give the following.

Lemma 5.3. Let $\hat{e_{1}}$ be a weight of $T$ which generates the subgroup $\hat{T}_{1} \cong S^{1}$ in $T$. Here the subgroup $S$ is as in Lemma 5.1. Then there is a diagonal basis of $T$ consisting of $\left\{\hat{e_{1}} \cup v_{1}, \ldots, \hat{e_{1}} \cup v_{N}\right\}$ where $\left\{v_{1}, \ldots, v_{N}\right\}$ is a diagonal basis for the hyperplane arrangement in $\operatorname{Lie}\left(S / \hat{T}_{1}\right)$ corresponding to the weights of the action of $S / \hat{T}_{1}$ on the tangent bundle to all components of the fixed point set of the action of $S / \hat{T}_{1}$ on $M_{1}$. (Note that here each $v_{k}$ is a basis for $\mathfrak{s} / \hat{\mathbf{t}_{1}}$.)

In the following theorem we consider a sum over all connected subgroups $S$ of the maximal torus $T$ of $G$ such that $\hat{T}_{1} \subset S$, with Lie algebra $\mathbf{s}$. We then consider a sum over all connected components $F$ of $M^{S}$ for which $T / S$ acts locally freely on $F$ (we refer to these as $F \in \mathcal{F}(S)_{\text {l.f. }}$ ).

Remark 5.4. In Example 7.3 we exhibit an example where a nontrivial group $T / S$ acts locally freely on $F \subset M^{S}$.

Theorem 5.5. (Residue formula in the general case) Let $\beta$ be an equivariant cohomology class of the form $\eta e^{\bar{\omega}}$ where $\eta \in H_{G}^{*}(M)$. Assume $c \in T$ is a regular value for $\Phi$.

Let $O B$ be the diagonal basis in $\mathcal{O B}(\triangle)$ corresponding to a total ordering of the elements in a hyperplane arrangement $\triangle=\triangle^{\prime}\left(S / \hat{T}_{1}\right) \cup\left\{\hat{e}_{1}\right\}$ for $\mathbf{s}$ (see Lemma 5.1). Here $\triangle^{\prime}\left(S / \hat{T}_{1}\right)$ is a hyperplane arrangement for $\mathbf{s} / \mathbf{t}_{1}$ which comprises all weights $\beta_{F, j}$ for the action of $S$ on the normal bundle to some component $F$ of the fixed point set of a connected subgroup $S$ of $T$. We sum over all such connected subgroups $S$. We have

$$
\begin{gathered}
\int_{\mu^{-1}(0) / G} \kappa\left(\eta e^{\bar{\omega}}\right)=\frac{n_{1}}{n_{0}^{\prime}|W|} \int_{\mu^{-1}(\mathbf{t}) / / T} \kappa\left(\eta \mathcal{D} e^{\bar{\omega}}\right)=\frac{n_{1}}{n_{0}^{\prime}|W|} \sum_{S \triangleleft T} \sum_{\sigma \in O B(S)} \sum_{F \in \mathcal{F}(S)_{1 . f .} .} \\
\frac{1}{\left|\mathbf{M} / \mathbf{M}_{\sigma}\right|} \int_{F / /(T / S)} \kappa_{T / S}\left\{\operatorname{Res}^{\sigma}\left(\mathcal{D}^{2}(X) \sum_{m \in \mathbf{M} / \mathbf{M}_{\sigma}} \frac{\eta(X) e^{(\mu(F)-m)(X)} e^{\omega_{F}}}{e_{F}(X) \prod_{\alpha \in \sigma}\left(1-e^{\alpha(X)}\right)}\right)\right\},
\end{gathered}
$$

where the notation $\operatorname{Res}^{\sigma}$ was introduced in Definition 1.8. Here $X \in \mathbf{t} \times \mathbb{C}$. The notation $e_{F}$ refers to the $S$-equivariant Euler class for the action of $S$ on the normal bundle to $F$ in $M$. Also, $n_{1}$ and $n_{0}^{\prime}$ were introduced in Proposition 3.5. 
Proof. We shall perform an induction reducing the rank of the group $T$ that acts on $M$, replacing $M$ by a component $M_{1}$ of $M^{\hat{T}_{1}}$, replacing $T$ by $T / \hat{T}_{1}$ and replacing $\eta$ by $\operatorname{Res}_{1} \eta$ in the notation of (1.8). Finally we reach a space $M_{i}$ and a group $T_{i}$ for which $T_{i}$ acts locally freely on $M_{i}$.

Let $\eta \in H_{T}^{*}(\widetilde{M})$; then by (3.2) combined with periodicity (in other words with Proposition 4.9) we have

$$
\begin{gathered}
\int_{\mu^{-1}(\mathbf{t}) / / T} \kappa_{T}\left(\mathcal{D} \eta e^{\bar{\omega}}\right) \\
=\sum_{\widetilde{M}_{1}} \int_{\mu^{-1}(\mathbf{t}) \cap \widetilde{M}_{1} \cap \mu_{T / \hat{T}_{1}}^{-1}(0) /\left(T / \hat{T}_{1}\right)} \kappa_{\left(T / \hat{T}_{1}\right)} \operatorname{Res}_{Y_{1}=0}\left(\frac{\mathcal{D} \eta e^{\bar{\omega}}}{\left(1-e^{Y_{1}}\right) e(\tilde{\nu})}\right) .
\end{gathered}
$$

Here $\hat{e_{1}} \in \mathbf{t}$ generates $\hat{T}_{1}$ and $\widetilde{M}_{1}$ is a component of $\widetilde{M} \hat{T}_{1}$ for which $0 \leq\left\langle\mu_{\hat{T}_{1}}\left(\widetilde{M}_{1}\right), \hat{e_{1}}\right\rangle$ $\leq\left\langle\hat{e_{1}}, \hat{e_{1}}\right\rangle$ (the set of such $\widetilde{M_{1}}$ is in bijective correspondence with the components $M_{1}$ of $\left.M^{\hat{T}_{1}}\right)$. Also $\tilde{\nu}$ is the normal bundle to $\widetilde{M}_{1}$ in $\mu^{-1}(\mathbf{t})$ and $e(\tilde{\nu})$ is its equivariant Euler class in $\mu^{-1}(\mathbf{t})$. These objects are in bijective correspondence with the corresponding objects $\nu$ and $e(\nu)$ in $M$, because when we restrict to $\mu^{-1}(\mathbf{t})$, the exponential map becomes a local diffeomorphism so the projection map from $\widetilde{M} \cap \mu^{-1}(\mathbf{t})$ to $M \cap \Phi^{-1}(T)$ is a covering map.

Recall from Remark 3.7 that the restriction of $\mu_{T}$ to $\mu^{-1}(\mathbf{t})$ is proper. Hence Proposition 4.9 can be applied. In the first paragraph of the proof of Lemma 4.4 (referring to Lemma 3.9) we establish the existence of regular values so that we can apply Proposition 4.9.

We assume by induction on $r$ that

$$
\int_{\left.\mu^{-1}(\mathbf{t})\right)^{\hat{T}_{1} / /\left(T / \hat{T}_{1}\right)}} \kappa_{r-1}\left(\mathcal{D} \eta e^{\bar{\omega}}\right)=\sum_{S^{\prime} \triangleleft T / \hat{T}_{1}} \sum_{\sigma^{\prime} \in O B\left(\triangle^{\prime}\left(S^{\prime}\right)\right)} \sum_{F^{\prime} \in \mathcal{F}\left(S^{\prime}\right)_{1 . f .}}
$$

$\frac{1}{\left|\mathbf{M}^{\prime} / \mathbf{M}_{\sigma^{\prime}}^{\prime}\right|} \operatorname{Res}^{\sigma^{\prime}} \int_{F^{\prime} / /(T / S)} \kappa_{T / S}\left\{\eta\left(X^{\prime}\right) \mathcal{D}\left(X^{\prime}\right) \sum_{m^{\prime} \in \mathbf{M}^{\prime} / \mathbf{M}_{\sigma^{\prime}}^{\prime}} \frac{e^{\left(\mu\left(F^{\prime}\right)-m^{\prime}\right)\left(X^{\prime}\right)} e^{\omega_{F^{\prime}}}}{e_{F^{\prime} \in \sigma^{\prime}}\left(1-e^{\alpha^{\prime}\left(X^{\prime}\right)}\right)}\right\}$.

where $O B\left(\triangle^{\prime}\left(S^{\prime}\right)\right)$ is a diagonal basis associated to a hyperplane arrangement $\triangle^{\prime}\left(S^{\prime}\right)$ in $\mathbf{t} / \hat{\mathbf{t}}_{1}$ consisting of all weights for the action of $S^{\prime}$ on the normal bundle to $F^{\prime}$ for all $F^{\prime} \in \mathcal{F}\left(S^{\prime}\right)_{\text {l.f. }}$ (see Lemma 5.1) and $e_{F^{\prime}}$ is the $\left(S^{\prime}:=S / \hat{T}_{1}\right)$-equivariant Euler class of the normal bundle in $\mu^{-1}(\mathbf{t})^{\hat{T}_{1}}$ to a component $F^{\prime}$ of the fixed point set of the action of $S^{\prime}$ on $\mu^{-1}(\mathbf{t})$, while $X^{\prime} \in \mathbf{t} / \hat{\mathbf{t}}_{1} \otimes \mathbb{C}$. Notice that $F^{\prime} \in \mathcal{F}\left(S^{\prime}\right)$ inherits a Hamiltonian action of $T / S^{\prime}$. If $T / S^{\prime}$ acts locally freely on $F^{\prime}$, then we cannot proceed further with the induction. Otherwise, continuing the induction we finally replace $F^{\prime}$ by the fixed point set $F^{\prime \prime} \subset F^{\prime}$ of a subgroup $S^{\prime \prime}$ for which $T / S^{\prime \prime}$ acts locally freely on $F^{\prime \prime}$. 
Remark 5.6. Note that if we define $S$ to be the pullback of the subgroup $S^{\prime}$ under the projection map $T \rightarrow T / \hat{T}_{1}$, it is clear that $T / S \cong T^{\prime} / S^{\prime}$ where $T^{\prime}=T / \hat{T}_{1}$.

Combining (5.3) with (5.4) we obtain

$$
\begin{gathered}
\int_{\mu^{-1}(\mathbf{t}) / / T} \kappa\left(\mathcal{D} \eta e^{\bar{\omega}}\right)=\sum_{\widetilde{M}_{1}} \sum_{S^{\prime} \triangleleft T / \hat{T}_{1}} \sum_{\sigma^{\prime} \in O B\left(\triangle^{\prime}\left(S^{\prime}\right)\right)} \sum_{F^{\prime} \in \mathcal{F}\left(S^{\prime}\right)_{1 . f .}} \frac{1}{\left|\mathbf{M}^{\prime} / \mathbf{M}_{\sigma^{\prime}}^{\prime}\right|} \operatorname{Res}^{\sigma^{\prime}} \operatorname{Res}_{Y_{1}=0} \\
\int_{F^{\prime} / /(T / S)} \kappa_{T / S}\left\{\eta\left(X^{\prime}\right) \mathcal{D}_{H}\left(X^{\prime}\right) \sum_{m^{\prime} \in \mathbf{M}^{\prime} / \mathbf{M}_{\sigma^{\prime}}^{\prime}} \frac{e^{\left(\mu\left(F^{\prime}\right)-m\right)\left(X^{\prime}\right)} e^{\omega_{F^{\prime}}}}{e_{F^{\prime}} e_{\widetilde{M_{1}}}\left(1-e^{Y_{1}}\right) \prod_{\alpha^{\prime} \in \sigma^{\prime}}\left(1-e^{\left.\alpha^{\prime}\left(X^{\prime}\right)\right)}\right\} .}\right.
\end{gathered}
$$

By Lemma 5.1 there is a bijective correspondence between the elements $\sigma^{\prime}$ in the diagonal basis $O B\left(\triangle^{\prime}\left(S^{\prime}\right)\right)$ and the elements $\sigma$ in a diagonal basis $O B(\triangle(S))$.

Note that $e_{F^{\prime}} e_{\widetilde{M_{1}}}$ is the equivariant Euler class $e_{F}^{\mathrm{t}}$ of the normal bundle to $F$ in $\mu^{-1}(\mathbf{t})$. The equivariant Euler class of the normal bundle to $F$ in $M$ is $e_{F}=e_{F}^{\mathbf{t}} \mathcal{D}$. Collecting these results we obtain (5.2).

By Lemma 5.1 the denominator of (5.5) is $e_{F} \prod_{\alpha \in \sigma}\left(1-e^{\alpha(X)}\right)$ for $\sigma$ the element of $O B(\triangle(S))$ formed from $\sigma^{\prime}$ and $\hat{e_{1}}$. This lemma also exhibits a bijective correspondence between $O B\left(\triangle^{\prime}\left(S^{\prime}\right)\right)$ and $O B(\triangle(S))$. By Lemma 5.2 $\left|\mathbf{M}^{\prime} / \mathbf{M}_{\sigma^{\prime}}^{\prime}\right|=\left|\mathbf{M} / \mathbf{M}_{\sigma}\right|$. In Remark 5.6 we have exhibited a bijective correspondence between connected subgroups $S$ containing $\hat{T}_{1}$ and connected subgroups $S^{\prime}$ of $T / \hat{T}_{1}$. The space $F^{\prime}$ is a component of $\left(\mu^{-1}(\mathbf{t})^{\hat{T}_{1}}\right)^{S^{\prime}}$ on which $T^{\prime} / S^{\prime}$ acts locally freely, so it is a component $F^{\prime}=F$ of $\mu^{-1}(\mathbf{t})^{S}$ on which $T / S$ acts locally freely. Finally the residue $\operatorname{Res}^{\sigma^{\prime}} \operatorname{Res}_{Y_{1}=0}$ is equal to $\operatorname{Res}^{\sigma}$.

This gives the result, using Lemmas 5.1, 5.2 and 5.3 to identify the diagonal bases.

Note that the finite sum

$$
\sum_{m \in \mathbf{M} / \mathbf{M}_{\sigma}} \frac{e^{-m(X)}}{\prod_{\alpha \in \sigma}\left(1-e^{\alpha(X)}\right)}
$$

parametrizes a class of different components of the fixed point set of the $T$ action in $\widetilde{M} \cap \mu^{-1}(\mathbf{t})$ which correspond to a given $F \subset M \cap \Phi^{-1}(T)$ (by specifying different values of the moment map $\mu$ which correspond to the same value of $\Phi(F))$. 


\section{Alekseev-Meinrenken-Woodward}

As we explained above, in [2] Alekseev, Meinrenken and Woodward gave a formula for intersection pairings of reduced spaces of a quasi-Hamiltonian $G$ space. In this section, we will relate our formula with Alekseev-MeinrenkenWoodward's by using Szenes' theorem (Theorems 1.17 and 1.13).

Let $\Lambda^{w}$ denote the weight lattice in $\mathbf{t}^{*}$. For $\lambda \in \Lambda^{w}$ let $\chi_{\lambda}$ denote the character of the corresponding irreducible representation $V_{\lambda}$.

Let $c \in Z(G)$. If $c$ is a regular value of $\Phi$ let

$$
\kappa: H_{G}^{*}(M) \rightarrow H^{*}\left(M_{\text {red }}\right)
$$

be the composition of the pull-back to the level set $H_{G}^{*}(M) \rightarrow H_{G}^{*}\left(\Phi^{-1}(c)\right)$ and the isomorphism $H_{G}^{*}\left(\Phi^{-1}(c)\right) \cong H^{*}\left(\Phi^{-1}(c) / G\right)$. (Here by analogy with (1.6) we define $M_{\text {red }}=\Phi^{-1}(c) / G$, cf. Theorem 1.3.) For any $\lambda \in \Lambda^{w}$, all fixed point manifolds $F \in \mathcal{F}(\lambda+\rho)$ (defined in the statement of Theorem 6.1) are contained in the preimage of the maximal torus $\Phi^{-1}(T)$. This follows by equivariance of $\Phi$. In fact, $F$ is a quasi-Hamiltonian $T$ space in the fixed point set of $S_{1}$ (where $S_{1}$ is the connected subgroup of $G$ generated by $\lambda+\rho$ ), with $\omega_{F}=\iota_{F}^{*} \omega$ as its symplectic form and $\Phi_{F}=\left.\Phi\right|_{F}$ as its moment map. Since $\Phi_{F}$ takes its value in $T$, we can compose with the map $T \rightarrow U(1), t \mapsto t^{\lambda+\rho}$ and this composition is denoted $\left(\Phi_{F}\right)^{\lambda+\rho}$. Note that $\left(\Phi_{F}\right)^{\lambda+\rho}$ is constant along $F$.

Theorem 6.1 (Alekseev-Meinrenken-Woodward [2]). Let $G$ be the direct product of a connected, simply connected Lie group and a torus, and $(M, \omega, \Phi)$ a compact quasi-Hamiltonian $G$-space. Suppose $\beta$ is a class of the form $\beta=\eta e^{\bar{\omega}}$ where $\eta \in H_{G}^{*}(M)$ and $\bar{\omega}$ was defined in (1.7). Then we have ([2], first equation $p$. 346, after the proof of Theorem 5.2)

$$
\begin{gathered}
\int_{M_{\mathrm{red}}} \kappa(\eta) \exp \left(\omega_{\text {red }}\right)= \\
\frac{k}{(v o l G)^{2}} \sum_{\lambda \in \Lambda_{+}^{w}}\left(d_{\lambda}^{2} \sum_{F \in \mathcal{F}(\lambda+\rho)}\left(\Phi_{F}\right)^{\lambda+\rho} \int_{F} \frac{\iota_{F}^{*} \eta(2 \pi i(\lambda+\rho))}{e_{F}(2 \pi i(\lambda+\rho))} e^{\omega_{F}}\right)
\end{gathered}
$$

where $k$ is the order of the principal stabilizer and $d_{\lambda}=\operatorname{dim} V_{\lambda}$. Here, $\mathcal{F}(\lambda+\rho)$ is the set of components of the zero locus of the vector field associated to $\lambda+\rho$. We use the notation $\Lambda_{+}^{w}$ to denote the set of weights in the fundamental Weyl chamber, i.e. the dominant weights.

Note that in [2], the sum over $\lambda \in \Lambda_{+}^{w}$ is actually over $\Lambda_{+}^{w} \subset \mathbf{t}_{+}$, the image in $\mathbf{t}$ of the intersection of the fundamental Weyl chamber in $\mathbf{t}^{*}$ with the weight lattice under the bijective map $\mathbf{t}^{*} \rightarrow \mathbf{t}$ given by the bi-invariant inner product. 
This map identifies the integer lattice in $\mathbf{t}$ with a sublattice of the image of the weight lattice.

To study the formula in the above theorem we need some tools from Lie group theory.

Theorem 6.2 (Weyl dimension formula, [10]). If the irreducible representation $V_{\lambda}$ of $G$ has highest weight $\lambda$, then its dimension is given by

$$
\operatorname{dim} V_{\lambda}=\prod_{\alpha \in R_{+}(G)} \frac{\langle\alpha, \lambda+\rho\rangle}{\langle\alpha, \rho\rangle},
$$

where $\langle\cdot, \cdot\rangle$ is the $W$-invariant inner product on $\mathbf{t}$ corresponding to the bi-invariant inner product on $\mathbf{g}$, and $R_{+}(G)$ are the positive roots of $G$.

Comparing Theorem 5.5 with Theorem 6.1: We will compare the formula (6.1) from [2] with our formula (5.2) in Theorem 5.5, when $G$ is a compact Lie group which is the product of a connected simply-connected Lie group with a torus.

Remark 6.3. In fact Theorem 6.1 is valid for arbitrary compact connected $G$ if the formula is interpreted in an appropriate way, using a finite cover $G^{\prime}$ of $G$ which is a product of a connected simply-connected group and a torus and an associated quasi-Hamiltonian $G^{\prime}$-space $M^{\prime}$ which is a finite cover of $M$. For simplicity the formula in [2] Theorem 5.2 is stated under the hypothesis that $G$ is the product of a connected simply connected Lie group and a torus. See Section 5.2 of [2].

Our proof invokes the hypothesis that $G$ is the product of a connected simplyconnected group and a torus to identify $\rho$ (half the sum of the positive roots of $G$ ) with an element in the weight lattice of $G$ (see Remark 6.4).

We will use Szenes' formula (Theorem 1.13) to relate (6.1) to our formula. We recast (6.1) (replacing $2 \pi i(\lambda+\rho)$ by $\lambda+\rho)$ as (6.2) below. We observe that $T$ acts on each component $F_{1}$ of the fixed point set of $\lambda+\rho$, so we can apply the Atiyah-Bott-Berline-Vergne localization theorem to replace

$$
\int_{F_{1}} \frac{\eta(\lambda+\rho)}{e_{F_{1}}(\lambda+\rho)}
$$

by

$$
\left.\sum_{F \subset\left(F_{1}\right)^{S}} \int_{F} \frac{\eta(X)}{e_{F}(X)}\right|_{X=\lambda+\rho}
$$

for any subgroup $S$ of $T$ containing the subgroup $S_{1}$ generated by $\lambda+\rho$ (using the fact that the equivariant Euler class is multiplicative so $e_{F}$ is the product of $e_{F_{1}}$ with the equivariant Euler class of the normal bundle to $F_{1}$ in $F$ ). We replace 
$F_{1}$ by $F$ and $S_{1}$ by $S$ and continue inductively until we reach a subgroup $S$ for which $T / S$ acts locally freely on $F$.

Remark 6.4. Recall that since $G$ is assumed to be the product of a connected simply connected group and a torus, $\rho \in \Lambda^{w}$. So we replace the sum over $\lambda+\rho$ by a sum over $\xi \in \Lambda_{0}^{w} \subset \mathbf{t}$, where $\Lambda_{0}^{w}$ is the set of strictly dominant weights, in other words those weights in $\mathbf{t}_{+}$not on the boundary of $\mathbf{t}_{+}$(not on any hyperplanes specified by one of the fundamental weights). We have used Weyl invariance of the sum to replace the sum over strictly dominant weights by a sum over the regular weights (those weights in $\mathbf{t}$ not on any hyperplanes specified by fundamental weights).

Hence we obtain

$$
\begin{gathered}
\int_{M_{\text {red }}} \kappa\left(\eta e^{\bar{\omega}}\right)=\frac{k}{|W|(V o l G)^{2}} \sum_{S \triangleleft T} \sum_{\lambda+\rho \in \Lambda_{0}^{w}(S)}\left(\operatorname{dim} V_{\lambda}\right)^{2} \\
\sum_{F \subset\left(M^{S}\right)_{\text {l.f. }}}\left(\Phi_{F}\right)^{\lambda+\rho} \int_{F} \frac{\eta(\lambda+\rho)}{e_{F}(\lambda+\rho)} e^{\omega_{F}},
\end{gathered}
$$

where $M^{S}$ is the fixed point set of the action of $S$ on $M$ and $e_{F}$ is the equivariant Euler class for the normal bundle to $F$ in $M$. Here $\left(M^{S}\right)_{\text {l.f. denotes the compo- }}$ nents $F$ of the fixed point set of $S$ acting on $M$ for which $T / S$ acts locally freely on $F$; this is indexed by the set $\mathcal{F}(S)_{\text {l.f. }}$. We subdivide $\Lambda_{0}^{w}$ into collections $\Lambda_{0}^{w}(S)$ of regular weights $\xi$ in the Lie algebra of a connected subgroup $S$ (those regular weights of $G$ which lie in $\mathfrak{s}$ ).

Now the formula (6.2) becomes

$$
\begin{gathered}
\int_{M_{\mathrm{red}}} \kappa\left(\eta e^{\bar{\omega}}\right)=\frac{k}{|W|(V o l G)^{2}} \sum_{S \triangleleft T} \sum_{\xi \in \Lambda_{0}^{w}(S)} \sum_{F \in \mathcal{F}(S)_{1 . \mathrm{f} .}} \\
\prod_{\alpha \in R_{+}(G)} \frac{\langle\alpha, \xi\rangle^{2}}{\langle\alpha, \rho\rangle^{2}} e^{\langle\mu(F), \xi\rangle} \int_{F} \frac{\eta(\xi)}{e_{F}(\xi)} e^{\omega_{F}} .
\end{gathered}
$$

(Here, for each $F$ we have chosen $\mu(F) \in \mathbf{t}$ for which $\Phi(F)=\exp \mu(F)$. The quantity $e^{\langle\mu(F), \xi\rangle}$ is well defined because $\xi \in \Lambda^{w}$.) Now we apply Szenes' theorem (separately for each $S$ ) to

$$
\mathcal{S}_{S}:=\sum_{\xi \in \Lambda_{0}^{w}(S)} \sum_{F \in \mathcal{F}(S)_{\text {l.f. }}} e^{\langle\mu(F), \xi\rangle} f_{F}(\xi)
$$


where

$$
f_{F}(X)=\prod_{\alpha \in R_{+}(G)} \frac{\langle\alpha, X\rangle^{2}}{\langle\alpha, \rho\rangle^{2}} \int_{F} \frac{\eta(X) e^{\omega_{F}}}{e_{F}(X)}
$$

for $X \in \operatorname{Lie}(T)$.

According to Szenes' theorem (Theorem 1.13),

$$
\begin{gathered}
\mathcal{S}_{S}=\sum_{\sigma \in O B\left(\triangle^{R}(S)\right)} \sum_{F \in \mathcal{F}(S)_{1 . \mathrm{f} .}} \\
\operatorname{Res}^{\sigma}\left(e^{\left\langle\mu(F), X_{S}\right\rangle} f_{F}\left(X_{S}\right) \frac{1}{\left|\mathbf{M} / \mathbf{M}_{\sigma}\right|} \sum_{m \in \mathbf{M} / \mathbf{M}_{\sigma}} \frac{e^{-m\left(X_{S}\right)}}{\prod_{\alpha \in \sigma}\left(1-e^{\alpha\left(X_{S}\right)}\right)}\right) .
\end{gathered}
$$

Here $X_{S} \in \operatorname{Lie}(S) \otimes \mathbb{C}$ and $\mathbf{M} \subset \mathbf{t}^{*}$ is the dual lattice to the lattice $\Lambda^{w}(S)$. We use the hyperplane arrangement $\triangle^{R}(S)$ given by the restriction of the simple roots of $G$ to the Lie algebra $\mathfrak{s}$ of $S$, and choose a diagonal basis $O B\left(\triangle^{R}(S)\right)$ for this hyperplane arrangement.

This gives for the final form of Theorem 6.1:

$$
\begin{gathered}
\int_{M_{\mathrm{red}}} \kappa(\beta) \exp \left(\omega_{\text {red }}\right)=\frac{k}{|W|(V o l G)^{2}} \sum_{S \triangleleft T} \mathcal{S}_{S} \\
=\frac{k}{|W|(V o l G)^{2}}\left(\prod_{\alpha \in R_{+}(G)} \frac{1}{\langle\alpha, \rho\rangle^{2}}\right) \sum_{S \triangleleft T} \sum_{F \in \mathcal{F}(S)_{1 . f .}} \sum_{\sigma \in O B\left(\triangle \triangle^{R}(S)\right)} \\
\frac{1}{\left|\mathbf{M} / \mathbf{M}_{\sigma}\right|}\left(\operatorname{Res}^{\sigma} \mathcal{D}^{2}\left(X_{S}\right) \sum_{m \in \mathbf{M} / \mathbf{M}_{\sigma}} \frac{e^{(\mu(F)-m)\left(X_{S}\right)}}{\prod_{\alpha \in \sigma}\left(1-e^{\alpha\left(X_{S}\right)}\right)} \int_{F} \eta(X) \frac{e^{\omega_{F}}}{e_{F}\left(X_{S}\right)}\right),
\end{gathered}
$$

where the notation $\operatorname{Res}^{\sigma}$ was introduced in Definition 1.10. Here $X_{S}$ is a variable in $\mathfrak{s} \otimes \mathbb{C}$.

Comparing (6.5) to our formula (5.2) in Theorem 5.5 the differences are:

(1) The presence in Theorem 5.5 of $\kappa_{T / S}$ and evaluation on $F / /(T / S)$ rather than $F$ as in Theorem 6.1. The identification between the two formulas follows because the following result is true:

Proposition 6.5. If a locally free action of $T / S$ on a quasi-Hamiltonian $T / S$ space $(F, \Phi)$ induces an action of $T$ on $F$, then for all $\eta \in H_{T}^{*}(F)$ and corresponding $\beta=\eta e^{\bar{\omega}}$

$$
\int_{F} \beta \circ i_{S}^{*}=\int_{F / /(T / S)} \kappa_{T / S} \beta
$$

where the inclusion $i_{S}: S \rightarrow T$ induces $i_{S}^{*}: H_{T}^{*}(F) \rightarrow H_{S}^{*}(F)$ (setting the variables $X$ corresponding to $T / S$ to zero) and both sides of (6.6) are in $H_{S}^{*}$, 
and $\kappa_{T / S}: H_{T}^{*}(F) \rightarrow H_{S}^{*}\left(\Phi^{-1}(e) /(T / S)\right.$ ) is the Kirwan map (in other words the restriction map to $\Phi^{-1}(e)$ composed with the isomorphism $H_{T / S}^{*}\left(\Phi^{-1}(e)\right) \cong$ $\left.H^{*}\left(\Phi^{-1}(e) /(T / S)\right)\right)$.

To prove this result it suffices to consider the case $S=\{e\}$ for which it reduces to verifying that

$$
\int_{\Phi^{-1}(e) \cap F / T} e^{\omega} \eta\left(X_{j}=c^{(j)}\right)=\left.\int_{F} e^{\omega} \eta\right|_{(X=0)} .
$$

(Here $c^{(j}$ is the first Chern class of the line bundle associated to the principal T-bundle

$$
T \rightarrow \Phi^{-1}(e) \mapsto \Phi^{-1}(e) / T
$$

by the $j$-th weight $T \rightarrow U(1)$.)

Proposition 6.5 is clear when the $c^{(j)}$ are 0 (i.e. when the bundle (6.8) is trivial). In general it is a special case of Theorem 5.2 of [2] (the case where the group $G$ is a torus and acts locally freely on $M$ ). We expect it may be possible to give a direct proof of this special case without relying on the full strength of Theorem 5.2 of [2]. For a related result, see [22].

(2) Differences between overall multiplicative constants

$$
\frac{k}{|W|(V o l G)^{2}}\left(\prod_{\alpha \in R_{+}} \frac{1}{\langle\alpha, \rho\rangle^{2}}\right) .
$$

and

Since by [6] Cor. 7.27

$$
\frac{n_{1}}{|W| n_{0}^{\prime}}
$$

$$
\frac{\operatorname{vol} G}{\operatorname{volT}}=\prod_{\alpha \in R_{+}(G)} \frac{1}{2 \pi\langle\alpha, \rho\rangle}
$$

where the volumes are taken with respect to the metrics on $G$ and $T$ given by the bi-invariant inner product $\langle\cdot, \cdot\rangle$, the equation $(6.9)$ is equal to

$$
\frac{(2 \pi)^{2 n+k}}{|W|(\operatorname{volT})^{2}}
$$

So the difference is $\frac{n_{1}}{|W| n_{0}^{\prime}}$ versus $\frac{(2 \pi)^{2 n}+k}{|W|(\text { volT })^{2}}$. This difference arises from the normalization of the Riemannian metrics used in (6.10).

(3) The hyperplane arrangement $\triangle^{R}(S)$ used in (6.5) is different from the hyperplane arrangement $\triangle(S)$ used in Theorem 5.5. If the weights at all components $F \in \mathcal{F}(S)$ are roots, the same hyperplane arrangement can be used. This is true for example in the case studied in [15]. 


\section{Applications}

Example 7.1: The main example for which the residue formula has been used previously to compute intersection numbers in reduced spaces of quasi-Hamiltonian spaces appears in [15], for which $M=G^{2 h}$ (where $G=S U(n)$ and $G$ acts by conjugation on $M$ ). In this example the fixed point sets of all vector fields $v_{\lambda}$ associated to weights $\lambda$ are equal to $M^{T}=T^{2 h}$.

Example 7.2: If $T$ is a torus, a standard example of a quasi-Hamiltonian $T$ space is $M=T \times T$, with the action of $T$ given by multiplication of the first copy of $T$. In this case $T$ acts freely on $M$. The moment map is the projection to the second factor $T$. The reduced space for this action is a point.

Example 7.3: An example due to C. Woodward [18] occurs when $G=S U(3)$, with rank two maximal torus $T$ for which $M$ is constructed starting with $G \times_{T} \mathbb{C} P^{2}$ by replacing three orbits $G / T$ (corresponding to the three fixed points of $T$ in $\mathbb{C} P^{2}$ ) by $G / U(1)_{j}($ for $j=0,1,2)$. Here $U(1)_{0} \subset T$ is generated by $\rho$ (where $\rho$ is half the sum of the positive roots, identified as an element of $\mathbf{t}$ using the biinvariant inner product) and $U(1)_{j}$ is generated by $c_{j} \rho$ (for $j=1,2$ ) where $c_{j}$ are nontrivial elements of the Weyl group (the cyclic permutations). The fixed point set $F_{j}$ of $U(1)_{j}$ acting on $M$ is symplectic, and is isomorphic to $S^{1} \times S^{1}$ with a multiple of the standard symplectic structure (this is the case treated in Example 7.2). The normal bundle of $F_{j}$ is trivial, and is isomorphic (as a representation of $\left.U(1)_{j}\right)$ to $\mathbb{C} \oplus \mathbf{t}^{\perp}$ (the direct sum of the trivial representation and the restriction from $T$ to $U(1)_{j}$ of the adjoint representation of $T$ on the orthocomplement $\mathbf{t}^{\perp}$ of $\mathbf{t}$ in $\mathbf{g}$, where $U(1)_{j}$ acts on $\mathbf{t}^{\perp}$ via $c_{j} \rho$.) Thus the Euler class is

$$
e_{F_{j}}\left(Y_{j}\right)=\widetilde{\gamma_{1}}\left(Y_{j}\right) \widetilde{\gamma_{2}}\left(Y_{j}\right)\left(\widetilde{\gamma_{1}}\left(Y_{j}\right)+\widetilde{\gamma_{2}}\left(Y_{j}\right)\right)
$$

where $\gamma_{1}$ and $\gamma_{2}$ are the simple roots of $S U(3)$ and the third positive root is $\gamma_{1}+\gamma_{2}$, and we have denoted by $\widetilde{\gamma}_{j}$ the restrictions of $\gamma_{j}$ to the Lie algebra of $U(1)_{j}$. Here we have introduced a nonzero element $Y_{j}$ of the Lie algebra of $U(1)_{j}$.

In this example $M^{T}=\emptyset$. The orbit type decomposition consists of two connected strata: the principal stratum where $G$ acts freely, and another stratum $M_{1}$ where the stabilizer is conjugate to $U(1)$.

By Theorem 5.5 the relevant subgroups $S$ are the $U(1)_{j}$ conjugate to $U(1)$; the group $T / S$ is also isomorphic to $U(1)$, and $T / S$ acts freely on the fixed point set $F_{j}$ of $U(1)_{j}$. So there is only one element in the hyperplane arrangement (which we denote by $\alpha_{j}$ ) and $\mathbf{M}_{\sigma}=\mathbf{M}$. So the expression in Theorem 5.5 reduces to

$$
\sum_{j=0}^{2} \int_{F_{j} / /\left(T / U(1)_{j}\right)} \kappa_{T / U(1)_{j}}\left\{\operatorname{Res}_{Y_{j}=0}\left(\frac{\mathcal{D}^{2}(X) \eta(X) e^{\mu\left(F_{j}\right)(X)} e^{\omega_{F_{j}}}}{e_{F_{j}}\left(1-e^{\alpha_{j}(X)}\right)}\right)\right\}
$$


where $Y_{j} \in \operatorname{Lie}\left(U(1)_{j}\right)$ is as above. In this example, $F_{j} \cong U(1) \times U(1)$ so $F_{j} / /\left(T / U(1)_{j}\right)$ is a point, as in Example 8.2. Thus the final map $\kappa_{T / U(1)_{j}}$ in our computation is

$$
\kappa_{U(1)}: H_{U(1)}^{*}(U(1) \times U(1)) \cong H^{*}(U(1)) \rightarrow H^{*}(\text { point })=H^{*}\left(\Phi^{-1}(e) / U(1)\right),
$$

which is the pullback map.

Acknowledgments: This article presents the main results of the Ph.D. thesis [20] of the second author, written under the supervision of the first author. The authors would like to acknowledge the helpful comments of Rebecca Goldin, Yael Karshon and Eckhard Meinrenken (who formed the committee of examiners for the second author's Ph.D. thesis). We wish to thank Anton Alekseev for pointing out that in an earlier version of Section 6 we had overlooked the distinction between a sum over the entire weight lattice and the dominant weights; the latter was used in Theorem 5.2 of [2]. We also wish to thank the referee for useful comments on an earlier version, and for alerting us to some relevant results in the literature.

\section{REFERENCES}

[1] A. Alekseev, A. Malkin, E. Meinrenken, Lie group valued moment maps J. Differential Geometry 48 (1998) 445-449.

[2] A. Alekseev, E. Meinrenken, C. Woodward, Group-valued equivariant localization. Inventiones Mathematicae 140 (2000) 327-350.

[3] A. Alekseev, E. Meinrenken, C. Woodward, Duistermaat-Heckman measures and moduli spaces of flat bundles over surfaces. Geometric and Functional Analysis 12 (2002) 1-31.

[4] A. Alekseev, E. Meinrenken, C. Woodward, The Verlinde formulas as fixed point formulas, J. Sym. Geo.1 (2001) 1-46.

[5] M.F. Atiyah, R.Bott, The moment map and equivariant cohomology. Topology 23 (1984) $1-28$.

[6] N. Berline, E. Getzler, M. Vergne, Heat Kernels and Dirac operators (Grundlehren \#298), Springer-Verlag, 1992.

[7] N. Berline, M. Vergne, Zéros d'un champ de vecteurs et classes caractéristiques équivariantes. Duke Math. J. 50 (1983) 539-549.

[8] M. Brion, M. Vergne, Arrangement of hyperplanes I: Rational functions and Jeffrey-Kirwan residue. Ann. Scient. Ec. Norm. Sup. 32 (1999) 715-741.

[9] M. Brion, M. Vergne, Arrangement of hyperplanes II: Szenes formula and Eisenstein series. Duke Math. J. 103 (2000) 279-302.

[10] J. J. Duistermaat, J.A.C. Kolk, Lie Groups. Springer-Verlag, 2002.

[11] R.F. Goldin, An effective algorithm for the cohomology ring of symplectic reductions, Geom. Anal. Funct. Anal. 12 (2002) 567-583.

[12] V. Guillemin, J. Kalkman, The Jeffrey-Kirwan localization theorem and residue operations in equivariant cohomology. J. reine angew. Math. 470 (1996) 123-142.

[13] L.C. Jeffrey Extended moduli spaces of flat connections on Riemann surfaces Math. Annalen 298 (1994) 667-692.

[14] L.C. Jeffrey, F.C. Kirwan, Localization for nonabelian group actions. Topology 34 (1995) 291-327. 
[15] L.C. Jeffrey, F.C. Kirwan, Intersection theory on moduli spaces of holomorphic bundles of arbitrary rank on a Riemann surface. Annals of Mathematics 148 (1998) 109-196.

[16] F. Kirwan, Cohomology of quotients in algebraic and symplectic geometry. Math. Notes vol. 31, Princeton Univ. Press, Princeton, NJ, 1985

[17] S.K. Martin, Symplectic quotients by a nonabelian group and by its maximal torus, preprint math.SG/0001002; Transversality theory, cobordisms, and invariants of symplectic quotients, preprint math.SG/0001001; Ann. Math., to appear.

[18] E. Meinrenken and C. Woodward, Chris Woodward's example, unpublished manuscript (2001).

[19] O. Plamenevskaya, A residue formula for $S U(2)$-valued moment maps, preprint math.DG/9906093; Can. Bull. Math., to appear.

[20] Joon-Hyeok Song, Intersection numbers in reduced spaces of q-Hamiltonian G-spaces, Ph.D. thesis, University of Toronto, 2004.

[21] A. Szenes, Iterated residues and multiple Bernoulli polynomials, International Mathematics Research Notices 18 (1998) 937-956.

[22] J. Weitsman, A Duistermaat-Heckman formula for symplectic circle actions, International Mathematics Research Notices 12 (1993) 309-312.

Lisa Jeffrey and Joon-Hyeok Song

Department of Mathematics

University of Toronto

Toronto, ON M5S 2E4, Canada

E-mail: jeffrey@math.toronto.edu

song@math.toronto.edu 